Portland State University

PDXScholar

$9-27-2021$

\title{
The Return to Anisotropy Across a Jet in Crossflow
}

Gregory P. Sakradse

Portland State University

Follow this and additional works at: https://pdxscholar.library.pdx.edu/open_access_etds

Part of the Aerodynamics and Fluid Mechanics Commons, and the Physics Commons Let us know how access to this document benefits you.

\section{Recommended Citation}

Sakradse, Gregory P., "The Return to Anisotropy Across a Jet in Crossflow" (2021). Dissertations and Theses. Paper 5811.

https://doi.org/10.15760/etd.7682

This Thesis is brought to you for free and open access. It has been accepted for inclusion in Dissertations and Theses by an authorized administrator of PDXScholar. Please contact us if we can make this document more accessible: pdxscholar@pdx.edu. 
The Return to Anisotropy Across a Jet in Crossflow

by

Gregory P Sakradse

A thesis submitted in partial fulfillment of the requirements for the degree of

\author{
Master of Science \\ in \\ Mechanical Engineering
}

Thesis committee:

Raúl Bayoán Cal, Chair

Gerald Recktenwald

Mark Weislogel

Portland State University

2021 


\begin{abstract}
With data from experiments on a jet of air emitting from an orifice flush with the floor of a wind tunnel providing a transverse flow, analysis is conducted to extract information about the state of anisotropy in the Reynolds stress tensor. Inflow velocities are modulated across two distinct turbulence intensity regimes while holding jet exit conditions constant, providing an opportunity to isolate effects of both jet to crossflow velocity ratio, $r$ and the effects of the turbulence carried by the crossflow. Anisotropy in the Reynolds stress tensor is examined through anisotropy invariant maps and evolution of the function $F$, combining the two independent invariants of the normalized anisotropy tensor, $b_{i j}$. A quaternion representation of the three dimensional rotations in the principle axes of the normalized anisotropy tensor is developed allowing for concise graphical representations of the eigen-matrix of $b_{i j}$. A component-wise view of the anisotropy tensor is presented showing the contributions of its individual components to the state of anisotropy. It is found that emanating into a crossflow which is itself largely anisotropic, a jet will develop a more isotropic core region as it bends over into the crossflow, this region persists beyond 10 jet diameters downstream of the exit. Some of the more isotropic turbulence diffuses beyond the commonly understood bounds of the jet and this effect is enhanced by more energetic turbulence in the crossflow.
\end{abstract}




\section{Acknowledgments}

I must express an immense gratitude for my advisor Raúl Bayoán Cal. Without his plucking me out of an undergraduate fluids class beginning what would become years of support, guidance, and good times none of this work would have been possible. To the members of the thesis committee, Mark Weislogel and Gerry Recktenwald I owe a great deal of gratitude. Both for agreeing to consider this work, and more importantly for the quality instruction and advice delivered over the my years in the department. The support of my colleagues in the wind energy and turbulence lab has been instrumental in my development as a researcher. Instrumental to this work was Graham Freedland who collected and pre-processed the data from the experiments. He also was generous enough to share some of his rich experience interpreting the flow providing a strong foundation for this analysis. Naseem Ali helped develop my understanding of Reynolds stress anisotropy and its role generally in turbulent dynamics, I have relied on his insights to develop all of the research work I have conducted and can't thank him enough for his patience and generosity. Long discussions with Sarah Smith have become my preferred way to develop MATLAB code. Without her debugging skills none of the analysis or graphics in this or all of my other work would be possible, she also makes for an amazing friend. Finally I

need to thank my family and dear friends who have gotten me through some very 
long days and nights, without their laughter and encouragement I would not have seen the end of this process. 


\section{Contents}

Abstract ...................... i

Acknowledgments .................... i i

List of Tables . . . . . . . . . . . . . . . . . . . vi vi

List of Figures . . . . . . . . . . . . . . . . . . vii

1 Introduction . . . . . . . . . . . . . . . . 1

2 A brief summary of turbulence modeling . . . . . . . . . . . 9

2.1 Brute Force Methods . . . . . . . . . . . . . . . . . . . . . . 10

2.2 Statistical Turbulence Modeling . . . . . . . . . . . . . . . . 11

3 Theory ......................... 19

3.1 Reynolds Stress Anisotropy . . . . . . . . . . . . . . . . . . . 19

3.1 .1 Principle Axes Rotation . . . . . . . . . . . . . . . . . . . . . . . . . . 22

3.1 .2 Background . . . . . . . . . . . . . . . . . . . . . . . . . . . . . . . . . . . . . . .

3.1 .3 Computation . . . . . . . . . . . . . . . . 26

4 Experiment . . . . . . . . . . . . . . . . . . . . 30

4.1 Apparatus/ Set Up . . . . . . . . . . . . . . . . . . . . . . . . . . . . . . . . . . .

4.2 Conditions . . . . . . . . . . . . . . . . . . . . . . . . . 32

$5 \quad$ Results \& Discussion . . . . . . . . . . . . . . . . . . . 34

5.1 Mean Flow . . . . . . . . . . . . . . . . . . . . . . 34

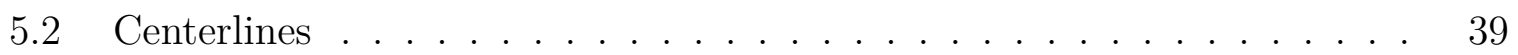

5.3 Reynolds Stress Anisotropy . . . . . . . . . . . . . . . . . . 43

5.4 Principle Axes Rotation . . . . . . . . . . . . . . 57

6 Conclusions ........................... 68

Bibliography ....................... 73 


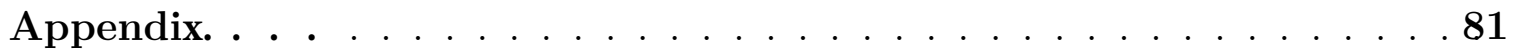

A Turbulence Viscosity Modeling _. . . . . . . . . . . . . 81

A.1 Zero Equation Models . . . . . . . . . . . . . . . . . . . . . . . 81

A.2 Kinetic Energy Models . . . . . . . . . . . . . . . . . 82 


\section{List of Tables}

3.1 Limiting states of realizable turbulence. . . . . . . . . . . . . 23

4.1 Conditions of considered cases. . . . . . . . . . . . . . . . 33

5.1 Residence octant of $\mathbf{n}$ based on the sign of its components. . . . . . . 57 


\section{List of Figures}

1.1 Diagram of the major vortex systems present in the jet in crossflow, figure directly from Fric and Roshko [20]. . . . . . . . . . . .

3.1 Anisotropy invariant map defines the bounds of realizable states of anisotropy. . . . . . . . . . . . . . . . . 22

4.1 Experimental setup . . . . . . . . . . . . . . . . . 30

5.1 Contours of (a) the horizontal component of mean velocity normalized by jet exit velocity, and (b) the vertical component of mean velocity normalized by jet exit velocity of passive grid cases. . . . . . . . . .

5.2 Contours of (a) the horizontal component of mean velocity normalized by jet exit velocity, and (b) the vertical component of mean velocity normalized by jet exit velocity of active grid cases. . . . . . . . . . . 36

5.3 Passive grid profiles of the horizontal component of mean velocity. . . 37

5.4 Passive grid profiles of the vertical component of mean velocity. . . . 37

5.5 Active grid profiles of the horizontal component of mean velocity. . . 38

5.6 Active grid profiles of the vertical component of mean velocity. . . . . 38

5.7 Centerlines from Smith and Mungal [21] and the improved centerlines plotted over contours of $\bar{v} \ldots$. . . . . . . . . . . . . . . . . . 40

5.8 Schematic describing centerline refinement algorithm. Velocities are rotated about the set of $\theta_{i}$ based on centerline $n-1$, resulting rotated

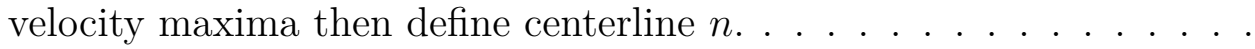

5.9 Locations of lines along which axial profiles are queried, each line is orthogonal to the centerline, colors correspond to the $x / d$ location at which the line intersects the centerline. Centerline and shear layers are shown to define the geometry of the jet. . . . . . . . . . .

5.10 Contours of anisotropy parameter, $F$ for passive grid cases, (a) and active grid cases $(\mathrm{b}) . \ldots \ldots \ldots \ldots$ 
5.11 Profiles of anisotropy parameter, $F$ along lines orthogonal to the centerline. Colorbars represent the distance from jet exit at which the profiled line crosses the centerline. Passive grid cases, (a) and active grid cases (b). . . . . . . . . . . . . . . .

5.12 Anisotropy invariant maps (AIM), or Lumley triangles of six axial profiles about the jet centerline for the passive grid cases (a) and active grid cases (b). . . . . . . . . . . . . . . .

5.13 Components of the passive grid cases anisotropy tensor $a_{i j}$ defined in equation (2.15). Diagonal tensor components normalized by the local maximum of the diagonal, and the off diagonal components similarly normalized by the maximum of the off diagonal component along each queried profile. . . . . . . . . . . . . . . .

5.14 Components of the active grid cases anisotropy tensor $a_{i j}$ defined in equation (2.15). Diagonal tensor components normalized by the local maximum of the diagonal, and the off diagonal components similarly normalized by the maximum of the off diagonal component along each queried profile. . . . . . . . . . . . . . . .

5.15 The flow broken up into 4 zones based on the shear layer and centerline, data shown under color map for example is $\left|\bar{u}_{i j}\right|$ of active grid $r=7.9 . \quad 58$

5.16 Octant definitions in which the residence of the rotation vector is defined. 58

5.17 Residence octants of the axis of rotation, $\mathbf{n}$ as described in (3.13). Passive grid cases (a) and active grid cases (b) . . . . . . . . . 59

5.18 Angle of rotation $\theta$ about $\mathbf{n}$, as described in (3.13). Passive grid cases (a) and active grid cases (b) . . . . . . . . . . . . 60

5.19 Counts of octant of $\mathbf{n}$ residence grouped by zone and normalized by the total number of queried points in each zone for both the passive grid (a) and active grid cases (b) . . . . . . . . . . . .

5.20 Angle of rotation $\theta$ colored binned by zone. Passive grid cases (a) and active grid cases $(\mathrm{b}) \ldots \ldots \ldots \ldots$ 
The jet in cross flow describes a canonical flow consisting of jet emitting a fluid at a large angle with a freestream. There are countless examples of such flows occurring naturally and in engineered environments. Laboratory scale buoyant jets in a crossflow mimic the scaled dynamics of volcanic plumes in the atmosphere [1]. Wastewater ejected into rivers and seas are sometimes subject to crossflow interactions which cause shore attachment [2]. The performance of modern gas turbine engines is largely due to the ability to run at very high temperatures, to achieve these high temperatures film cooling systems are employed [3]. Film cooling is achieved by the ejection of bleed air through ports on the leading edge of turbine blades [4]. The cooling performance achieved by film cooling allows for gas temperatures to exceed allowable metal temperatures, however certain topologies of crossflow turbulence can encourage separation of the cool jet fluid from the boundary layer causing a detrimental effect on performance and potential damage to the turbine[5].

The jet and the freestream can be of the same species, as is the case in ships bow thrusters, where a jet of accelerated water is expelled from an orifice on the side of a ship to act as a source of thrust to enact a turning moment on the ship to ease maneuvering [6]. In other applications, such as direct fuel injection, the jet and crossflow are of different species. Studying jets of water into a freestream of 
air, Eslamian et al. [7] found that the jet momentum ratio, $q=\rho_{j} V_{j}^{2} / \rho_{\infty} V_{\infty}^{2}$ is the primary variable associated with jet trajectory, while turbulence intensities in the jet and the freestream seem to impact liquid atomization, mixing, and droplet dispersion. In areas of unsedimented sea floor, sea water can seep into the earths crust and interact with magma chambers, the then superheated water is buoyantly driven back up through the sea floor in what is known as hydrothermal vent, resulting in a plume [8]. Hydrothermal vents are known to be rich sources of minerals, and locally create unique and biomass rich ecosystems in contrast to the typical sea floor ecosystem, but are also critical sources of dissolved minerals in the oceans at large as a result of the flow driven transport mechanisms [9][10]. The transport of dissolved and particulate minerals emitted from hydrothermal vents throughout the ocean is found to be highly dependent on the entrainment flow and vortex systems of the plume, features common to all jets in crossflow [11].

Formal study of the jet in cross flow begins with interest in the dispersion of pollutants from industrial chimneys. Bosanquet and Pearson [12] use eddy diffusion theory to develop a model for coal particle dispersion from a smoke stack, concluding that at large distances away the particle concentration at ground level is largely independent of stack height. A series of wind tunnel experiments, again focusing on dispersion of pollutants from smoke stacks result in empirical relationships for the vertical and horizontal distances at which the jet becomes "substantially horizontal". When normalized by stack exit diameter each of these distances depends only on the ratio of stack emission velocity to the crossflow velocity[13]. The ratio of jet emission velocity to crossflow velocity, often labeled $r=V_{J} / V_{\infty}$, is equivalent to the momentum ratio $q$ defined above in cases where the jet fluid and the crossflow fluid are of equal densities. Both $r$ and $q$ depending on configuration are the critical non-dimensional parameters 
found in literature used to classify jets in crossflow, and in many cases to collapse data such as trajectory, and width [14] [15]. Recent studies suggest that scaling on only $r$ and and the jet diameter, $d$ are not sufficient to collapse some configurations [16]. Arguments have been made that a more appropriate scaling must also consider the boundary layer thickness, an approach that shows success at collapsing trajectories [17].

Keffer and Baines [18] use both hot wire anemometry and imaging of an oil-vapor / nitrogen smoke seeded jet in a wind tunnel to explore scaling. They employ what is termed a natural coordinate system based on coordinates representing the centerline, and locus of maximum velocity respectively, along with a third coordinate perpendicular to the others. Using the natural coordinate system, the authors seek to collapse of various turbulence quantities as functions of a local length scale only to demonstrate independence from crossflow velocity. Collapse to a common curve is achieved for mean velocities, though higher order moments do not collapse suggesting the the interaction with the crosswind creates complex dynamics preventing similarity.

In another wind tunnel study of a jet of air into air Andreopoulos and Rodi [19] take measurements with a triple wire hot wire probe and are thus able to collect three components of velocity simultaneously. They report a complete turbulent kinetic energy (TKE) budget along traversed profiles for a jet with a relatively small jet to crosswind velocity ratio, $r=0.5$. Directly over the jet exit and at the windward edge of the jet core the TKE is large, production terms dominate the budget, and are balanced largely by advection. The production terms at this point due to normal stresses are significantly higher than those due to the shearing stresses. Profiles 
four jet diameters downstream of the exit show relatively small TKE in the lee side region below the jet, the total TKE increases within the jet where production due to shearing stress becomes dominant, and is balanced by diffusion and dissipation. Above the jet on the windward edge, the balance shifts to advection and diffusion dominant.

A detailed description of the vortex dynamics in the wake region of the jet in crossflow is found in Fric and Roshko [20]. Using both a smoke seeded jet, and smoke wire seeding of the crossflow, detailed photographs of the system of coherent structures observed in the wake of the jet are presented. Comparing the jets wake to that of a cylinder, the characteristic open wake seen in the lee side of a flow around a cylinder is not seen in the jet, the crossflow closes in around the jet core resulting in an adverse pressure gradient on the boundary layer. Boundary layer vorticity is then ejected and entrained in the jet causing tornado like coherent structures, wake vortices between the wall and the lee side of the jet to be advected downstream as depicted in figure 1.1. It is also suggested that the eruption events, and thus the appearance of the connected coherent structures occur at regular frequencies in a narrow range of jet to crossflow ratios around $r \sim 4$.

Exploring mixing Smith and Mungal [21] employ planar laser-induced florescence, an imaging technique which allows for accurate measurement of of the concentration of a scalar field. By modulating nozzle diameter, the authors are able to maintain a constant $r d$ length scale across velocity ratios from $r=5$ to $r=200$. In their analysis, a multi region scaling is proposed, very near the jet exit quantities scale with $d$, allowing for observed variation in $r$, in the near field the jet scales with $r d$, in the region where $x / r^{2} d>0.2$, the scaling transitions to $r^{2} d$. They propose that the 


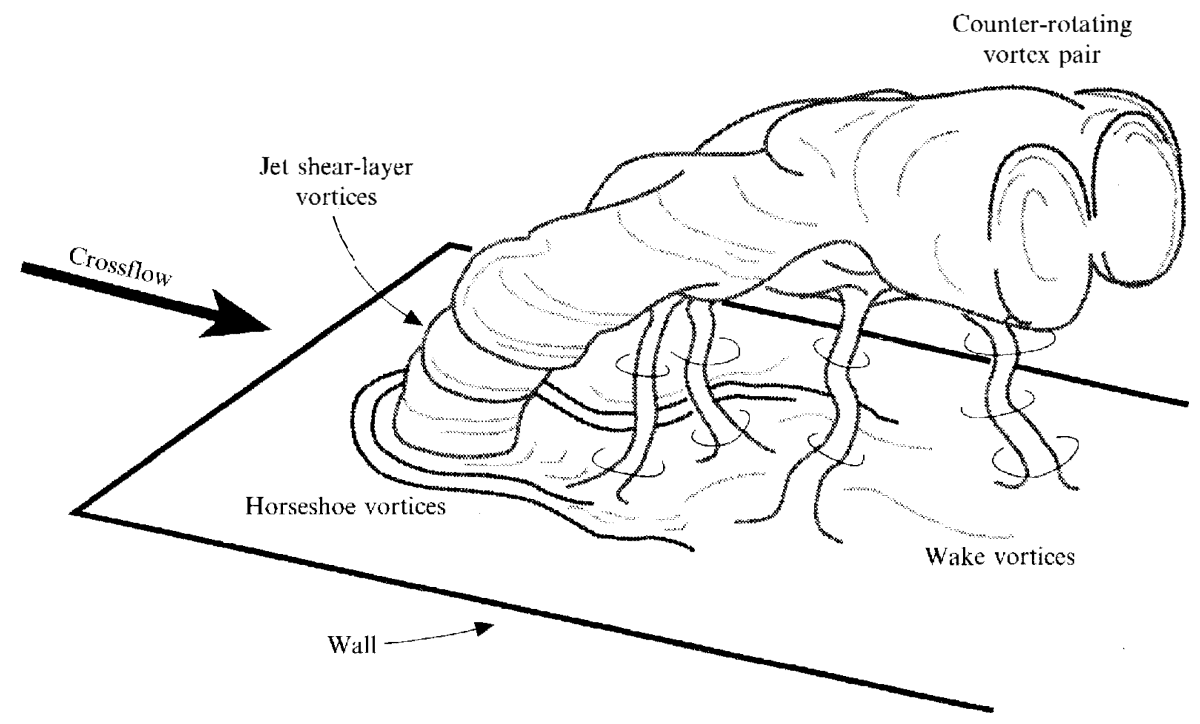

Figure 1.1: Diagram of the major vortex systems present in the jet in crossflow, figure directly from Fric and Roshko [20].

different scaling regimes correspond to the area of formation of the counter rotating vortex pair, the development of the counter rotating vortex pair, and finally a region where the counter rotating vortex pair is fully developed.

Additional exploration into the mixing dynamics is found in Shan and Dimotakis [22], experiments conducted in a water tunnel with a seeded jet of water emitted from the top. Scalar concentration data are collected via laser induced florescence at a velocity ratio of $r=10$ with jet Reynolds numbers ranging from $1 \times 10^{3}$ to $20 \times 10^{3}$. Results of scalar separation distance show less variance in vertical separations compared to horizontal separations at small distances, a feature not present for larger separations this suggests that the small scales of the flow are anisotropic, with a preferred vertical direction.

The typical experimental arrangement studied, and the arrangement considered in this work, consists of an jet emitting from a round orifice flush with a planar surface 
subjected to a crossflow with an established boundary layer and far from any walls [23][24][25]. There are various other configurations that can be described as a jet in crossflow such as a jet emitting from an orifice in a tube within a tube, such is the cases in the use of central venous catheters used in hemodyalysis. In such an application, the jet emitted through a port on the side of the catheter within the vein. Interactions occur with both the crossflow and the wall of the superior vena cava, resulting in complex flow dynamics not seen in the freestream configurations. The coupled interactions can potentially damage the wall of the vein [26].It is critical to consider the implications of flow geometry when interpreting experimental results.

There is much interest in the development and refinement of models of turbulent jets in crossflow ranging in complexity from simply predicting trajectory to the simulation of the fully 3 dimensional flow field. Early models relied on scaling arguments and experimentally determined constants for parameters such as trajectory or scalar concentration. Integral models reduced the need for experimental results to achieve solutions for such quantities, but often at the expense of accuracy [27]. With advances in computational resources many authors apply turbulent viscosity models to the flow such as $k-\mathcal{E}$ closure which is described in some detail in appendix 6 . Early results from such modeling attempts typically fail to mimic behavior seen in experiments [28]. Interested in accelerating the development process of turbine film cooling design, Claus [29] compares the results of a $k-\mathcal{E}$ turbulence model to experimental data. Large discrepancies are shown in even basic mean velocity profiles, the isotropy assumption in the Reynolds stresses modeled is blamed for the model to be of "limited usefulness"[29]. A more recent study on $k-\omega$ models compares the model output to experimental data of a supersonic jet into a transonic crossflow. The model 
shows an over predicted velocity deficit in addition to inaccurate strength and location of the counter rotating vortex pair. Beyond this the model fails to differentiate the natural length scales Reynolds stress tensor components [30].Further discussion on why such models may be inappropriate for the complexities of the jet in crossflow is discussed in section 2. The four common Reynolds stress transport models in use are compared to experiments on a jet into ambient, with much better agreement than the eddy viscosity models attempted before suggesting that models that bypass the Boussinesq hypothesis could preform better in shear flows.

The jet in crossflow is a perfect combination of ubiquity and complexity. Because the flow is seen in many applications it is well studied and there is much demand for accurate modeling. As it is also so complex there are still active questions to explore about the fundamental physics of the flow, correlated to the amount of active questions, it is a particularly difficult flow to accurately model. Particularly, as discussed above the readily available and often utilized $k-\mathcal{E}$ often fails to capture the mean velocity field. Recently, Ray et al. [31] employed a Bayesian optimization scheme to tune the constants in the $k-\mathcal{E}$ model, while they were able to better predict the mean flow, and even the normal components of the Reynolds stress tensor, their model failed to identify any Reynolds shear stress. It is of course possible to preform direct numerical simulation (DNS) on the flow field with good results such as those obtained by Muppidi and Mahesh [32]. Large eddy simulation (LES) is very capable of extracting the dominant features of the flow field [33]. These computationally expensive methods are not ideal for many applications, much of the work in the modeling of volcanic plumes relies on integral models built on sparse measurements as initial conditions, working on scales inconceivable to DNS [34]. There is much room in the field of turbulence modeling to bring the prediction accuracy higher for lightweight simulations. 
A great deal of current work explores replacing the closure models for the Reynolds stress tensor in the Reynolds averaged Navier Stokes equations with machine learning algorithms an approach that has the capability to revolutionize the way RANS simulations are conducted[35]. New parameterizations of turbulent flows are relevant in this context, Wu et al. [36] uses a quaternion parameterization of the anisotropy tensors eigenvectors in a machine learning model in order to predict the perturbations required to to correct RANS simulated Reynolds stress tensors, a similar quaternion parameterization method is employed in this work to qualitatively describe the position of the principle axes of the anisotropy tensor.

It is in the context of an evolving turbulent modeling space that the Reynolds stress anisotropy tensor of the jet in cross flow is studied here. The development of imaginative approaches to the persistent modeling problem relies on an understanding of the physics of the flow, and in complex flows such as the jet in crossflow there are seemingly infinite views into the physics. Presented here is a summary of classical framework of why Reynolds stress anisotropy is relevant to the questions of turbulence modeling, and with it a brief derivation of the relevant equations found in section 2 . The basis of the analysis built upon the classical framework and as briefly discussed above, a rather unique approach through quaternion representations of rotating axes is described in section 3. Results are given, first a characterization of the mean flow, followed by two views into the anisotropy of the Reynolds stress tensor, a qualitative assessment of a quaternion representation of the principle axes of said Reynolds stress anisotropy tensor, and finally a component wise view of said tensor. The work is then concluded with conclusions, found in section 6 . 
The general governing equations which completely describe a the motion of a fluid, based on the laws of classical mechanics and formalized by Claude-Louis Navier in 1821, and later apparently independently by George Gabriel Stokes in 1850, are known as Navier-Stokes equations [37]. Applied to an incompressible, Newtonian fluid the Navier-Stokes equations based on the conservation of mass and momentum yield the form,

$$
\begin{gathered}
\frac{\partial u_{i}}{\partial x_{i}}=0 \\
\frac{\partial u_{i}}{\partial t}+u_{j} \frac{\partial u_{i}}{\partial x_{j}}=-\frac{1}{\rho} \frac{\partial p}{\partial x_{j}}+\nu \frac{\partial^{2} u_{i}}{\partial x_{j} \partial x_{j}} .
\end{gathered}
$$

The vector $u_{i}$ represents the instantaneous velocity field, $\rho$ the fluid density, $p$ the pressure, and $\nu$ is the kinematic viscosity. While regarded as the foundation of modern fluid mechanics, the Navier-Stokes equations did not become relevant to the 
study of fluid dynamics until the 1880s with the exploration of turbulent flows by Reynolds and Boussinesq [38]. As a consequence of the non-linear convection term, equations (2.1) and (2.2) can be solved analytically only in the simplest of model flows such as laminar channel flow, Couette flow, and Poiseuille flow in a pipe [39]. These analytical solutions to the Navier-Stokes equations are however of little practical use as they require generally non-physical assumptions to reduce the equations to a form where a solution exists and are of course incapable of describing a turbulent flow field.

\subsection{Brute Force Methods}

It is possible to solve (2.1) and (2.2) numerically, they make up a closed system of differential equations, solutions exist. Direct numerical simulation (DNS) solves (2.1) and (2.2) simultaneously in three dimensions across time, ideally capturing all time and spacial scales, and is thus perfectly suited to generating solutions to turbulent flows which prohibit analytical solutions [40]. Difficulties arise when attempting to design direct numerical simulations as a direct consequence of the potential instabilities in solutions to the Navier-Stokes equations. The left hand side of equation (2.2) has the effect of introducing sharp gradients and discontinuities to potential solutions, while the left hand side smooths and causes solutions to decay. The Reynolds

number, $R e=U L / \nu$ describes the balance between the chaotic material derivative, and the terms on the left, as Re increases the flow will become more violent and numerical treatments of such flows must be very stable. 
Early work in DNS was conducted at the National Center for Atmospheric Research and is summarized in Fox and Lilly [41], the authors identify the principle hurdle to the brute force method of arriving at solutions to (2.1) and (2.2), as the immense range of scales at which turbulent dynamics take place. In order to resolve the entire spectrum of scales at which the motion responsible for the transfer of energy in a turbulent flow take place, simulations must be run on a computational mesh that is large enough to capture the largest turbulent scales, generally dictated by the flow geometry, simultaneously the resolution of the mesh must be smaller than the Kolmogorov length scale $\eta=\left(\nu^{3} / \mathcal{E}\right)^{1 / 4}$ where the dissipation of energy occurs [42]. Scale separation in turbulent flows is a function of re the number of mesh points

required to simulate a flow is approximated by $N_{x y z}=(L / \Delta)^{3} \approx 1.7 R e^{9 / 4}$, where $T$

$L$ is the domain size, $\Delta$ the mesh separation, and $r e_{T}$ the Reynolds number of the large scale turbulence, as a consequence in order to triple the Reynolds number of a simulation an increase of approximately 10 times as many mesh points is required $[43]$.

The needs of many modelers are not to resolve the energy dissipative scales of the turbulence, but rather to understand a subset of bulk flow variables. When this is the case, a statistical approach to modeling may be sufficient to answer many practical questions about a flow.

\subsection{Statistical Turbulence Modeling}

The foundations of the statistical treatment of turbulence is based on the decomposition of the turbulent velocity field into a mean and fluctuating component introduced 
in the 1885 paper of Reynolds [44]. What is now known as Reynolds decomposition separates the instantaneous velocity $u_{i}$, into a mean component $\overline{u_{i}}$, and a component which fluctuates about the mean $u_{i}^{\prime}$, such that

$$
u_{i}=\overline{u_{i}}+u_{i}^{\prime}
$$

When (2.3) is applied to (2.1)and (2.2), the result averaged and manipulated, the Reynolds averaged Navier-Stokes equations (RANS) are the result,

$$
\begin{gathered}
\frac{\partial \overline{u_{i}}}{\partial x_{i}}=0 \\
\frac{\partial \overline{u_{i}}}{\partial t}+\overline{u_{j}} \frac{\partial \overline{u_{i}}}{\partial x_{j}}=-\frac{1}{\rho} \frac{\partial \bar{p}}{\partial x_{i}}+\frac{\partial}{\partial x_{j}}\left(2 \overline{s_{i j}}-\overline{u_{i}^{\prime} u_{j}^{\prime}}\right)
\end{gathered}
$$

where $\bar{p}$ is the mean pressure and $\overline{s_{i j}}=\frac{1}{2}\left(\frac{\partial \overline{u_{i}}}{\partial x_{j}}+\frac{\partial \overline{u_{j}}}{\partial x_{i}}\right)$ the mean strain rate, an additional term $\overline{u_{i}^{\prime} u_{j}^{\prime}}$ appears as a result of the averaging process. The additional correlation in $(2.5)$ is a consequence of averaging non-linear acceleration term, while not a viscous stress stress as the $\overline{s_{i j}}$ term, it shares the same units and has the effect of acting as a stress on the mean motion it is thus termed the Reynolds stress tensor and is responsible for the transfer of energy between the fluctuating motion to the mean motion [45]. 
In order to find solutions to the RANS equations, additional equations are typically sought in an effort to describe the evolution of the $\overline{u_{i}^{\prime} u_{j}^{\prime}}$ term. An exact transport equation for the Reynolds stresses can be constructed by applying a first moment to (2.2) based on fluctuating velocities $u_{i}^{\prime}$ and $u_{j}^{\prime}$, the process consists of multiplying $\left(u_{i}^{\prime}+u_{j}^{\prime}\right)$ by each term in (2.2) and performing a Reynolds decomposition on the instantaneous quantities and averaging the result [40]. After much manipulation the resulting equation takes the form

$$
\frac{\partial \overline{u_{i}^{\prime} u_{j}^{\prime}}}{\partial t}+\overline{u_{k}} \frac{\partial \overline{u_{i}^{\prime} u_{j}^{\prime}}}{\partial x_{k}}=P_{i j}-\frac{\partial}{\partial x_{k}} T_{i j k}-\mathcal{\varepsilon}_{i j}+\Pi_{i j}
$$

The left had side of (2.6) is the convective derivative of the Reynolds stress tensor, $\overline{u_{i}^{\prime} u_{j}^{\prime}}$. This is balanced by the following terms,

$$
\begin{gathered}
P_{i j}=-\overline{u_{i}^{\prime} u_{k}^{\prime}} \frac{\partial \overline{u_{j}}}{\partial x_{k}}-\overline{u_{j}^{\prime} u_{k}^{\prime}} \frac{\partial \overline{u_{i}}}{\partial x_{k}} \\
T_{i j k}=\overline{u_{i}^{\prime} u_{j}^{\prime} u_{k}^{\prime}}+\frac{1}{\rho}\left[\overline{p^{\prime} u_{i}^{\prime}} \delta_{j k}+\overline{p^{\prime} u_{j}^{\prime}} \delta_{i k}-\nu \frac{\partial \overline{u_{i}^{\prime} u_{j}^{\prime}}}{\partial x_{k}}\right], \\
\mathcal{E}_{i j}=2 \nu \overline{\frac{\partial u_{i}^{\prime}}{\partial x_{k}}} \frac{\partial u_{j}^{\prime}}{\partial x_{k}} \\
\Pi_{i j}=\frac{1}{\rho}\left(\frac{\overline{p^{\prime} \partial u_{i}^{\prime}}}{\partial x_{j}}+\frac{\overline{p^{\prime} \partial u_{j}^{\prime}}}{\partial x_{i}}\right)
\end{gathered}
$$

$P_{i j}$ is the production of Reynolds shear stress from the mean flow, $T_{i j k}$, the flux, $\mathcal{E}_{i j}$ the dissipation, and $\Pi_{i j}$ the pressure rate of strain correlation. If one were to seek transport equations for higher order moments of fluctuating velocities, such as 
a transport equation for the triple correlation in the $T_{i j k}$ term of (2.6) the right hand side will always contain a correlation tensor of order $n+1$, where $n$ is the moment order of the transport equation constructed, closure is impossible with this approach $[46]$.

As exact closure of the RANS equations and their moments is not possible, various approaches have been developed to relate the Reynolds stresses to mean flow quantities. A family of these methods is based on the turbulent viscosity hypothesis originally published in 1877 by Boussinesq [47], which in its modern form gives a linear relationship between the Reynolds stress tensor and mean strain rate as

$$
\overline{u_{i}^{\prime} u_{j}^{\prime}}=\frac{2}{3} k \delta_{i j}-\nu_{T}\left(\frac{\partial \overline{u_{i}}}{\partial x_{j}}+\frac{\partial \overline{u_{j}}}{\partial x_{i}}\right)
$$

where $k$ is the turbulence kinetic energy, and $\nu_{t}$, turbulent viscosity, sometimes known as eddy-viscosity. Interestingly, the original formulation of the Boussinesq hypothesis is presented almost a decade earlier than the first appearance of the RANS equations, Boussinesq recognizes an apparent motion dependent component of the stress in turbulent flows independent of the notion of Reynolds stresses as they are now understood [48].

The turbulent viscosity is generally a function of space and time, to ensure dimensional consistency. It is defined as the ratio of the square of a length scale and a time scale, 


$$
\nu_{T}(x, t)=l^{2} / \tau
$$

A vast number of approaches have been proposed to specify $l$ and $\tau$, some examples of which are provided in appendix 6, all fall under the umbrella term of turbulence viscosity models [49]. Implicit in all turbulence viscosity models is the underlying assumption of (2.8), that the physics of the turbulence is analogous to the physics of the molecular processes governing the viscous stress law in a Newtonian fluid described by the relation between the strain rate and the viscous stress tensor $[50]$

$$
-\left(\tau_{i j}+\bar{p} \delta_{i j}\right) / \rho=-2 \nu \overline{s_{i j}}
$$

The eddy viscosity theory requires that the Reynolds stress tensor maintain a linear relationship with the mean strain rate tensor, which is to say that any anisotropy in $\overline{u_{i}^{\prime} u_{j}^{\prime}}$ is a direct local result of anisotropy in the strain rate tensor $\overline{s_{i j}}=\frac{\partial \overline{u_{i}}}{\partial x_{j}}+\frac{\partial \overline{u_{j}}}{\partial x_{i}}[48]$. A classic example of the failing of this hypothesis are the contraction experiments of Uberoi [51]. The effect of an axial contraction is to impose, and then remove an extensive axial strain and compressive lateral strains on the flow [50]. Initially nearly isotropic turbulence becomes more anisotropic in the presence of an mean gradient, this anisotropy in $\overline{u_{i}^{\prime} u_{j}^{\prime}}$ persists downstream of the contraction in the absence of mean 
gradients [51]. Were $(2.8)$ to hold, any anisotropy in $\overline{u_{i}^{\prime} u_{j}^{\prime}}$ would vanish as $\overline{s_{i j}} \rightarrow 0$, which is not the case [52].

RANS models need not rely on (2.8) to characterize the $\overline{u_{i}^{\prime} u_{j}^{\prime}}$ in (2.5). Second order closure schemes seek to model terms of (2.6) directly, and are capable of decoupling anisotropy in $\overline{u_{i}^{\prime} u_{j}^{\prime}}$ from that of $\overline{s_{i j}}$.

To develop second order turbulence models, one must express the terms from the right hand side of (2.6) in terms of the Reynolds stress tensor, $\overline{u_{i}^{\prime} u_{j}^{\prime}}$, its gradients, $\partial \overline{u_{i}^{\prime} u_{j}^{\prime}} / \partial x_{k}$, mean velocity gradients, $\partial \overline{u_{i}} / \partial x_{j}$, dissipation rate, $\mathcal{E}$, and viscosity $\nu$ [53]. It is common in the development of second order closure schemes to consider the case of homogeneous anisotropic turbulence, in this configuration $\partial \overline{u_{i}} / \partial x_{j}$ and $\partial T_{i j k} / \partial x_{k}$ are zero yielding a simplified version of (2.6) [54],

$$
\frac{\partial \overline{u_{i}^{\prime} u_{j}^{\prime}}}{\partial t}=\Pi_{i j}-\varepsilon_{i j}
$$

This relationship in homogeneous turbulence can be further simplified by noting that with high Reynolds numbers the dissipation rate tensor becomes nearly isotropic and can be represented as [53],

$$
\mathcal{E}_{i j}=\frac{2}{3} \mathcal{E} \delta_{i j}
$$


where $\mathcal{E}$ is the scalar dissipation. Additionally further insight into the pressure rate of strain relationship can be gained by examining the Reynolds decomposition of the Poisson equation for the fluctuating pressure [50],

$$
\frac{1}{\rho} \frac{\partial^{2} p^{\prime}}{\partial x_{i} \partial x_{i}}=-2 \frac{\partial \overline{u_{i}}}{\partial x_{j}} \frac{\partial u_{j}^{\prime}}{\partial x_{i}}-\frac{\partial^{2}}{\partial x_{i} \partial x_{j}}\left(u_{i}^{\prime} u_{j}^{\prime}-\overline{u_{i}^{\prime} u_{j}^{\prime}}\right) .
$$

The two terms on the right hand side of (2.13) are known as the rapid pressure term, $p^{\prime(r)}$, and the slow pressure term, $p^{\prime(s)}$. The rapid pressure, the first term, vanishes in homogeneous turbulence, the second term, the slow pressure has no dependence on mean gradients and only contains information about the role of the turbulence on the fluctuating pressure [55]. Equation (2.11) for high Reynolds number homogeneous turbulence can now be written with the pressure rate of strain term from only the slow pressure component

$$
\frac{\partial \overline{u_{i}^{\prime} u_{j}^{\prime}}}{\partial t}=\Pi_{i j}^{(s)}-\mathcal{E}_{i j}=\frac{2}{3} \varepsilon \delta_{i j} .
$$

As the dissipation rate tensor is now considered isotropic, the only possible anisotropy in (2.14) comes directly from the Reynolds stress tensor itself. It is convenient to decompose the Reynolds stress tensor into a deviatoric tensor [56] by subtracting the isotropic portion. This yields, 


$$
a_{i j}=\overline{u_{i}^{\prime} u_{j}^{\prime}}-\frac{2}{3} k \delta_{i j}
$$

with $k$, the turbulence kinetic energy, half the trace of $\overline{u_{i}^{\prime} u_{j}^{\prime}}$. Normalizing $a_{i j}$ by its trace gives the Reynolds stress anisotropy tensor [57],

$$
b_{i j}=\frac{\overline{u_{i}^{\prime} u_{j}^{\prime}}}{2 k}-\frac{1}{3} \delta_{i j}
$$

The general forms of second order closure schemes seek to define functional relationships which describe the evolution of $\Pi_{i j}$ which are dependent on $b_{i j}$ only [58]. 


\subsection{Reynolds Stress Anisotropy}

Being a traceless and symmetric tensor, $b_{i j}$ can be completely characterized by two invariants $I I$, and $I I I$, which are related to the variables $\eta$ and $\xi$ by

$$
\eta^{2}=-I I / 3, \quad \xi^{3}=I I I / 2
$$

The invariants of $b_{i j}$ expressed in the variables $\eta$ and $\xi$ expressing the second and third invariants respectively are

$$
\begin{gathered}
b_{i i}=0, \\
6 \eta^{2}=b_{i i}^{2}=b_{i j} b_{j i}, \\
6 \xi^{3}=b_{i i}^{3}=b_{i j} b_{j k} b_{k i} .
\end{gathered}
$$


The second invariant takes positive values and defines the degree of anisotropy in $b_{i j}$, with zero representing a state of isotropy. The third invariant can take both positive and negative values, with positive values indicating one-component turbulence, and negative, two-component turbulence.

The state of anisotropy can be equivalently described via the eigenvalues of the normalized anisotropy tensor. An eigenvalue decomposition of the symmetric tensor $b_{i j}$ yields a set of eigenvectors corresponding to the principle axes of its vector space,

$$
b_{i j}=\Sigma_{i j} \lambda_{i} \Sigma_{i j}^{-1} .
$$

Due to the first invariant, $b_{i i}$, being zero, the sum of the resulting 3 eigenvalues is zero, leaving one eigenvalue as a combination of the others [50]. In its principle axes, $b_{i j}$ can be expressed as

$$
\hat{b}_{i j}=\left(\begin{array}{ccc}
\lambda_{1} & 0 & 0 \\
0 & \lambda_{2} & 0 \\
0 & 0 & -\lambda_{1}-\lambda_{2}
\end{array}\right) .
$$

The second and third invariants can thus be expressed as functions of the eigenvalues of the anisotropy tensor 


$$
\begin{gathered}
\eta^{2}=\frac{1}{3}\left(\lambda_{1}^{2}+\lambda_{1} \lambda_{2}+\lambda_{2}^{2}\right), \\
\text { and } \xi^{3}=-\frac{1}{2} \lambda_{1} \lambda_{2}\left(\lambda_{1}+\lambda_{2}\right) .
\end{gathered}
$$

The values that the invariants can take are bounded by realizability conditions of the Reynolds stress tensor. The realizability conditions require non-negative energies, and bound the cross correlations between velocity components by the magnitude of their autocorrelations [59]. The result of the realizability conditions on the Reynolds stress tensor is to bound the possible values that the anisotropy tensor invariants and eigenvalues can take while still representing a physical system [52]. When expressed in terms of $\eta$ and $\xi$ the bounds of realizability form the anisotropy invariant map (AIM), commonly referred to as the Lumley triangle, all physically possible values of the invariants fall within the boundaries of the triangle of figure 3.1 and the limiting values are defined in table 3.1 [57].

The limiting state of anisotropy forming the two component line along the top of the Lumley triangle from 2 component axisymmetric to purely one component anisotropy represents a state of anisotropy where one of the Reynolds stresses is always zero. In contrast the origin of the Lumley triangle is a purely 3 dimensional isotropy, where all components of the Reynolds stress tensor are of equal weight, and the anisotropy tensor is null. A functional relationship between the purely two dimensional and purely 3 dimensional states of anisotropy is given by 


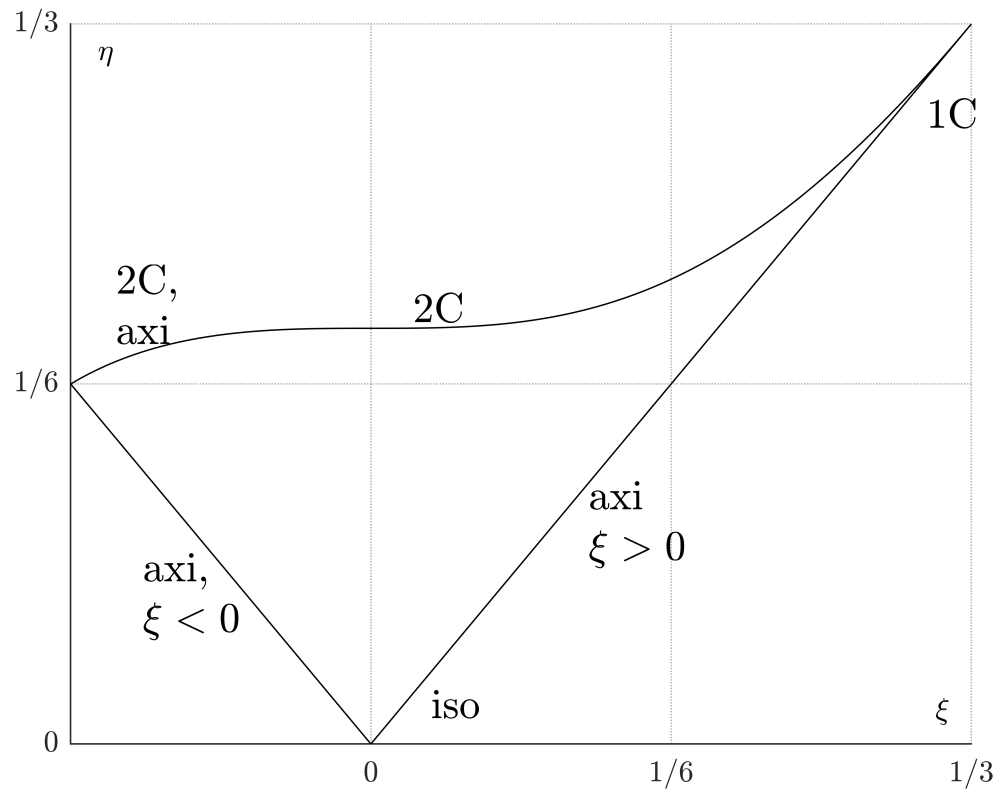

Figure 3.1: Anisotropy invariant map defines the bounds of realizable states of anisotropy.

$$
F=1-27 \eta^{2}+54 \xi^{3} .
$$

The function $F$ ranges between 0 in purely two-dimensional anisotropy, and 1 in purely isotropic turbulence [57].

\subsubsection{Principle Axes Rotation}


Table 3.1: Limiting states of realizable turbulence.

\begin{tabular}{lcc}
\hline State & Invariants & Eigenvalues \\
\hline Isotropic & $\eta=\xi=0$ & $\lambda_{1}=\lambda_{2}=0$ \\
Two-component axisymmetric & $\eta=\frac{1}{6}, \xi=-\frac{1}{6}$ & $\lambda_{1}=\lambda_{2}=\frac{1}{6}$ \\
One-component & $\eta=\xi=\frac{1}{3}$ & $\lambda_{1}=\frac{2}{3}, \lambda_{2}=$ \\
& $\eta=\xi$ & $-\frac{1}{3}$ \\
Axisymetric (one large & & $-\frac{1}{3} \leq \lambda_{1}=$ \\
eigenvalue) & $\eta=-\xi$ & $0 \leq \lambda_{2}=\lambda_{2} \leq$ \\
$\begin{array}{l}\text { Axisymetric (one small } \\
\text { eigenvalue) }\end{array}$ & $\frac{1}{6}$ \\
Two-component & $\eta=\left(\frac{1}{27}+2 \eta^{3}\right)^{\frac{1}{2}}$ & $\lambda_{1}+\lambda_{2}=\frac{1}{3}$ \\
\hline
\end{tabular}

\subsubsection{Background}

The eigen-decomposition of the anisotropy tensor in equation (3.5) yields both a vector of eigenvalues, $\lambda_{i}$, and a matrix whose columns are their associated eigenvectors, $\Sigma_{i j}$. As the anisotropy tensor, $b_{i j}$ is symmetric the eigenvectors in $\Sigma_{i j}$ are orthonormal and describe the set of principle axes for $b_{i j}$. The principle axes of the isotropic portion of the Reynolds stress tensor, $\frac{2}{3} k \delta_{i j}$ will simply fall on the axes of the coordinate system on which it is defined. The anisotropy tensor however will reside on principle axes made up of 3 unit vectors which can be described in relation to the global coordinate system.

Early work in the examination of the principle axes of the Reynolds stress tensor can be found in Hinze and Builtjes [60], studying the anisotropy in the Reynolds stress tensor in decaying grid turbulence in the absence of a mean gradient. Experimental data in two-dimensions collected downstream of a turbulence generating 
grid is used to define a single angle representing the rotation of the $x$-component of the principle axes of the Reynolds stress tensor with the $x$-component of the global coordinate system. They observe this angle decreasing, and far enough downstream the $x$-component of the principle axes is coincident with the $x$-component of the global coordinate system. This behavior is consistent with the other measurements of return-to-isotropy in grid generated turbulence.

As the current data provide Reynolds stress tensors in three dimensions, an examination of the orientation of the principle axes must made via parameters which can describe rotations in 3-dimensions. Much work has been done in the fields of controls and robotics towards implementing robust definitions for rigid body rotations in three dimensions [61] [62] [63] [64]. The set of eigenvectors contained in $\Sigma_{i j}$ can be interpreted as a rotation matrix, $\mathbf{R}$ with properties:

$$
\operatorname{det} \mathbf{R}= \pm 1 \quad \text { and } \quad \mathbf{R}^{-1}=\mathbf{R}^{T}
$$

Due to the nature of the eigen-decomposition in equation (3.5), $\Sigma_{i j}$ satisfies the properties of a rotation matrix, and thus is a member of the special orthogonal group $S O(3)$, and can be directly used to describe the rotation of the principle axes [65]. While the rotation matrix fully parameterizes any arbitrary rotation in three dimensions, it contains 9 elements, of which only 3 are independent [66]. A set of three or more parameters is necessary to completely characterize rotations in 3-dimensional space, common parameterizations in the study of rigid body kinematics include Euler 
parameters, Rodrigues parameters, Euler angles, and Cayley-Klein parameters, with Euler angles the most commonly used [67].

Euler angles describe three ordered rotations about the global coordinate axes which fully defines the attitude of a rigid body after an arbitrary rotation [68]. Application of Euler angles to describe the principle axes of the Reynolds stress tensor can be found in Hamilton and Cal [69], where examining the wakes of a counter-rotating wind turbine arrays with stereo PIV data, Euler angles are used to describe the spatial evolution of orientation of the principle axes within the wakes. A major downside in the use of Euler angles in the description of rotation is inherit in their formulation, singularities exist at angles $\beta=n \pi$ for $n \in \mathbb{Z}$, this is known as gimbal lock, leaving the Euler angles undefined for these angles [70].

A singularity free parameterization of three dimensional rotations can only be achieved by utilizing a fourth parameter to describe the rotation in $\mathbb{R}^{3}$, this leads to the use of quaternions [71]. A four dimensional extension of imaginary numbers, quaternions were originally formalized in the 19th century by Hamilton [72], it was not until the late 20th century that the use of quaternions to represent rotations began being adopted as a more computationally stable alternative to Euler angles [73]. They were used to define the error in the star tracker module in the navigation system aboard the space shuttle [74]. The quaternion parameterization has been gaining favor in the fields of computer graphics, controls, and robotics for its concise mapping of rotations in $\mathbb{R}^{3}[75]$. 


\subsubsection{Computation}

Quaternions are defined in four dimensional space, $\mathbb{H}$, spanned by a real axis and three additional orthogonal imaginary axes, the principle imaginaries are the unit pure quaternions* $^{\mathbf{i}}, \mathbf{j}, \mathbf{k}$ which define the three imaginary axes in $\mathbb{H}[76]$. A quaternion in $\mathbb{H}$ is composed of a real part, $e_{0}$, and an imaginary part $q_{1} \mathbf{i}+q_{2} \mathbf{j}+q_{3} \mathbf{k}$ to form $\underline{\mathbf{q}}=q_{0}+q_{1} \mathbf{i}+q_{2} \mathbf{j}+q_{3} \mathbf{k}[72]$.

To relate a 4 -dimensional quaternion in $\mathbb{H}$ to a rotation in $\mathbb{R}^{3}$ it is necessary to invoke Eulers theorem of rigid body rotations, the general displacement of a rigid body with one point fixed is a rotation about some axis, every rotation can be described by an angle $\theta$ to describe the rotation, and a vector $\mathbf{n}$ about which the rotation occurs [68]. Eulers axis-angle parameterization of rotations can be expressed directly by the quaternion as,

$$
\underline{\mathbf{q}}=\cos \frac{\theta}{2}+\sin \frac{\theta}{2} \mathbf{n}
$$

which can alternately be expressed in vector notation,

\footnotetext{
${ }^{*}$ Pure quaternions are quaternions with a zero real part.
} 


$$
\underline{\mathbf{q}}=\left[\begin{array}{c}
\sin \frac{\theta}{2} \\
\cos \frac{\theta}{2} \mathbf{n}
\end{array}\right]=\left[\begin{array}{l}
q_{0} \\
q_{1} \\
q_{2} \\
q_{3}
\end{array}\right]
$$

The real part of the quaternion is related to the sine of one half the angle or rotation, $q_{0}=\sin \theta / 2$ and the imaginary part, forms the vector axis of rotation, scaled by the cosine of one half the angle of rotation [76],

$$
\left[\begin{array}{l}
q_{1} \\
q_{2} \\
q_{3}
\end{array}\right]=\sin \frac{\theta}{2}\left[\begin{array}{l}
n_{x} \\
n_{y} \\
n_{z}
\end{array}\right]
$$

By way of a direction cosine matrix any rotation matrix $\mathbf{R}$ can be expressed directly in terms of the components of a unit quaternion expressing the same rotation $[77]$

$$
\mathbf{R}=\left(\begin{array}{ccc}
q_{0}^{2}+q_{1}^{2}-q_{2}^{2}-q_{3}^{2} & 2\left(q_{1} q_{2}-q_{0} q_{3}\right) & 2\left(q_{1} q_{2}+q_{0} q_{2}\right) \\
2\left(q_{1} q_{2}+q_{0} q_{3}\right) & q_{0}^{2}-q_{1}^{2}+q_{2}^{2}-q_{3}^{2} & 2\left(q_{2} q_{3}-q_{0} q_{1}\right) \\
2\left(q_{1} q_{3}-q_{0} q_{2}\right) & 2\left(q_{2} q_{3}+q_{0} q_{1}\right) & q_{0}^{2}-q_{1}^{2}-q_{2}^{2}+q_{3}^{2}
\end{array}\right)
$$

with the addition of the unitary condition, 


$$
q_{0}^{2}+q_{1}^{2}+q_{2}^{2}+q_{3}^{2}=1,
$$

the components of the unit quaternion, $\mathbf{q}$ can be calculated. As the quaternions provide a double mapping of rotations in $\mathbb{R}^{3}$ there will always be two possible solutions to the equation defined in (3.14) and (3.15), by convention the solution yielding a nonnegative $q_{0}$ is chosen which bounds the rotation space to rotations of an angle, $0 \leq \theta \leq 180$ [78]. While solutions exist for (3.14) and (3.15), problems can occur in their calculation with floating point arithmetic, as multiple divisions are necessary, very small values in the rotation matrix can cause singularities in the calculation of the quaternion components, several algorithms have been proposed to address errors and failure in calculation with various levels of success [79][80].

Recent work in the development of a robust rotation matrix to quaternion algorithm extends the rotation matrix from $\mathbb{R}^{3}$ to $\mathbb{R}^{4}$, utilizing a matrix factorization developed by Cayley [81]. Rotation in $\mathbf{R}$ can be considered as the product of isoclonic rotations in two directions. From this the quaternion components can be directly calculated by the norms of the rows or columns of matrix resulting from factorization in $\mathbb{R}^{4}$ [82]. The resulting quaternion components are calculated as, 


$$
\begin{aligned}
\left|q_{0}\right| & =\frac{1}{4} \sqrt{\left(r_{11}+r_{22}+r_{33}+1\right)^{2}+\left(r_{32}-r_{23}\right)^{2}+\left(r_{13}-r_{31}\right)^{2}+\left(r_{21}-r_{12}\right)^{2}} \\
\left|q_{1}\right| & =\frac{1}{4} \sqrt{\left(r_{32}-r_{23}\right)^{2}+\left(r_{11}-r_{22}-r_{33}+1\right)^{2}+\left(r_{21}+r_{12}\right)^{2}+\left(r_{31}+r_{13}\right)^{2}} \\
\left|q_{2}\right| & =\frac{1}{4} \sqrt{\left(r_{13}-r_{31}\right)^{2}+\left(r_{21}+r_{12}\right)^{2}+\left(r_{22}-r_{11}-r_{33}+1\right)^{2}+\left(r_{32}+r_{23}\right)^{2}} \\
\left|q_{3}\right| & =\frac{1}{4} \sqrt{\left(r_{21}-r_{12}\right)^{2}+\left(r_{31}+r_{13}\right)^{2}+\left(r_{32}+r_{23}\right)^{2}\left(r_{33}-r_{11}-r_{22}+1\right)^{2}}
\end{aligned}
$$

where $r_{i j}$ are the components of a rotation matrix. Following convention $q_{0}$ is assigned a positive sign while the remaining components $q_{1},, q_{2}$, and $q_{3}$ are assigned the signs of $\left(r_{32}-r_{23}\right),\left(r_{13}-r_{31}\right)$, and $\left(r_{21}-r_{12}\right)$ respectively [82]. 


\subsection{Apparatus/ Set Up}

The experiment utilizes the closed loop wind tunnel at Portland State University. The test section of the wind tunnel is $5 \mathrm{~m}$ long with a cross section of $0.8 \mathrm{~m} \times 1.2$ $\mathrm{m}$, and features a contraction ratio of 9:1. The tunnel is capable of producing free stream velocities ranging from $2 \mathrm{~m} / \mathrm{s}$ to $20 \mathrm{~m} / \mathrm{s}$. An active turbulence generating grid is installed at the inlet to the test section. Active grids are an extension of more traditional passive turbulence generating grids and have been found to effectively produce homogeneous and nearly isotropic turbulence with turbulence intensities stronger than those produced by their passive counterparts [83].

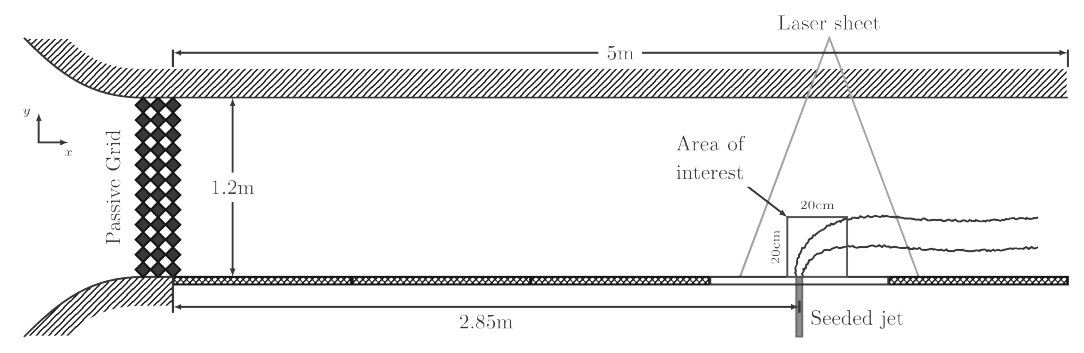

Figure 4.1: Experimental setup

The active grid used in this experiment consists of 6 horizontal and 7 vertical rods with $10 \mathrm{~cm}$ square winglets affixed. Each of the thirteen rods are driven with an in- 
dependent stepper motor. An in house LabView script controls the both the velocity and direction of each motor via random number generation with 13 individual seeds. The experiments are conducted either with the active grid actuated, which is subsequently referred to as active grid (AG) or configured such that each rod is positioned with its winglets aligned with the flow, a configuration referred to as passive grid (PG) which emulates a large mesh passive turbulence generating grid.

The jet apparatus consists of a round pipe exit, $9.525 \mathrm{~mm}$ in diameter mounted flush with the floor of the wind tunnel $2.85 \mathrm{~m}$ downstream from the turbulence generating grid. The pipe is fed air at a regulated pressure from a high pressure source. After regulation, the air-stream is mixed with a seeding stream of atomized diethylhexyl sebacate (DEHS) generated with a LaVision aerosol generator which produces a polydisperse neutrally buoyant aerosol with fluid particles between 0.2 $\mu \mathrm{m}$ and $1.0 \mu \mathrm{m}$. The air-stream and seeding stream are allowed $0.85 \mathrm{~m}$ to mix in the pipe before the jet exit.

Measurements are taken with a stereo particle image velocimetry (SPIV) system. Two LaVision 4MP ImagerProX CCD cameras are used to capture images, the cameras are mounted at an interior angle of $\approx 30^{\circ}$ to each other and calibrated to capture images along a plane perpendicular to the inflow and oriented at the centerline of the jet exit. The cameras are calibrated using a standard two plane calibration plate designed for use with the LaVision DaVis PIV software, the camera focus area defines the measurement plane which has dimensions of $0.2 \mathrm{~m} \times 0.2 \mathrm{~m}$. A schematic showing the orientation of the measurement plane to the jet as situated in the wind tunnel can be found in figure 4.1. The cameras are synced to collect images simultaneously, for each snapshot of data a total of four images are collected at two times separated by 
a time interval of $250 \mu \mathrm{s}$. A $532 \mathrm{~nm} \mathrm{Nd:Yag} \mathrm{laser} \mathrm{producing} 1200 \mathrm{~mJ}$ in $4 \mathrm{~ns}$ bursts is used to illuminate the measurement plane during the collection of each image pair. The laser sheet is focused on a plane at the center of the jet and parallel to the crossflow establishing the experimental $x y$ plane. The maximum laser sheet thickness, measured along the floor of the wind tunnel is is $\sim 2 \mathrm{~mm}$.

Each snapshot, containing four images is processed in the LaVision DaVis software system. The DaVis software utilizes an iterative fast Fourier transform correlation algorithm to determine the displacement of tracer particles between successive image pairs with the interrogation window shrinking between iterations. The interrogation window is set to $32 \times 32$ pixels for the first two passes, and reduced to $16 \times 16$ pixels for the second two passes, a $50 \%$ spatial overlap is set for the interrogation windows. This algorithm results in a vector field spatial resolution of $0.1 \mathrm{~mm}$. The uncertainty in the correlation is calculated in the software with an algorithm described in Wieneke [84], and is reported as less than $5 \%$ for all cases.

\subsection{Conditions}

Eleven experiments are run, five in the passive grid configuration and six running the active grid, for this analysis a subset of three passive gird and three active grid cases are chosen form the larger set. Inflow velocity $U_{\infty}$, is varied while mean jet exit velocity $V_{j}$, is held fixed at $26.0 \mathrm{~m} / \mathrm{s}$ yielding a jet exit Reynolds number with a length scale of jet diameter, $R e_{J}=V_{J} d_{J} / \nu=1.82 \times 10^{4}$. The velocity ratio $r=V_{J} / U_{i}$ has been used since the earliest investigations into jets in cross flow and is used here to differentiate cases [23]. The variation in inflow velocity is achieved by 
Table 4.1: Conditions of considered cases.

\begin{tabular}{cccccc}
\hline & $r$ & $U_{\infty}$ & $R e_{\infty}$ & $I$ & Frames \\
\hline \multirow{3}{*}{ Passive } & 5.09 & 5.11 & $3.23 \times 10^{3}$ & 0.07 & 2400 \\
& 7.53 & 3.45 & $2.18 \times 10^{3}$ & 0.04 & 2400 \\
& 13.97 & 1.86 & $1.76 \times 10^{3}$ & 0.13 & 2500 \\
\hline \multirow{3}{*}{ Active } & & & & & \\
& 5.88 & 4.42 & $2.79 \times 10^{3}$ & 0.17 & 3000 \\
& 7.92 & 3.28 & $2.07 \times 10^{3}$ & 0.23 & 3000 \\
& 12.52 & 2.08 & $1.315 \times 10^{3}$ & 0.16 & 3000
\end{tabular}

setting a constant fan speed driving the wind tunnel, for this reason the comparable AG and PG cases do not have exactly the same inflow mean velocity as a larger portion of the energy from the mean flow is lost to turbulence in the active grid cases. In varying the inflow condition while holding the jet flow constant, the effect of the inflow can be isolated from any effects that may occur from the variation of the jet.

Table 4.1 lists the experimental conditions for all the cases considered, the inflow Reynolds number uses jet diameter as a length scale for $R e_{\infty}$, mean inflow velocity is simply the average velocity along a vertical profile above the boundary layer and without the jet. $I$ is turbulence intensity, calculated as $I=\sqrt{k} /\left|U_{i}\right|$, where $k=$ $1 / 2\left(\overline{u^{\prime} u^{\prime}}+\overline{v^{\prime} v^{\prime}}+\overline{w^{\prime} w^{\prime}}\right)$, the turbulent kinetic energy. The number of snapshots, or frames, is increased in the active grid cases, due to the higher turbulence intensities longer data may be required for convergence. 


\subsection{Mean Flow}

Figures 5.1 and 5.2 show contours of the mean velocities in both the horizontal $\bar{u}$, and vertical $\bar{v}$ directions for the passive and active grid sets, respectively. Data are presented across the entire interrogated flow field to show the dramatic differences in jet trajectory across the range of velocity ratios considered. It should be noted that direct comparison between cases should be contained to the region $\sim<10 d$ downstream

of the jet exit as this is the region where the core of the jet is lost above the bounds of the interrogation region in the highest considered velocity ratio.

Figures 5.3 and 5.4 show vertical profiles of horizontal and vertical mean velocities respectively at stations between $x / d=0.25$ and $x / d=10$ for the passive grid cases. Profiles of mean velocities in the active grid set are similarly displayed in figures 5.5 and 5.6. The profiles show the full range of velocity ratios available so as to provide a more clear picture of the evolution of the mean velocity fields with velocity ratio.

The horizontal component of mean velocity $u$, shown in the passive grid cases in figure 5.1a shows an acceleration beyond the cross flow velocities on the windward 

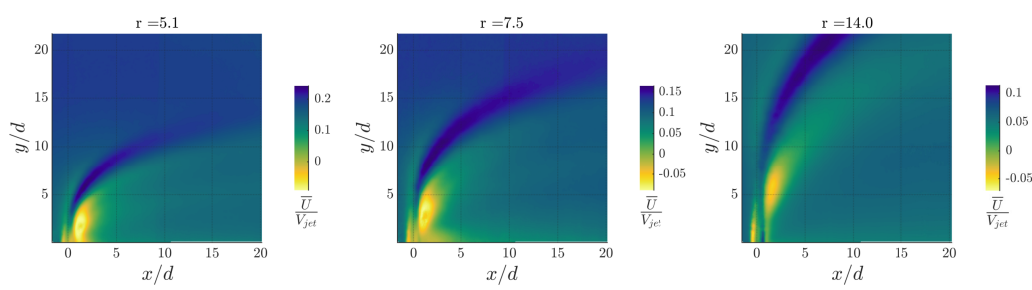

(a)
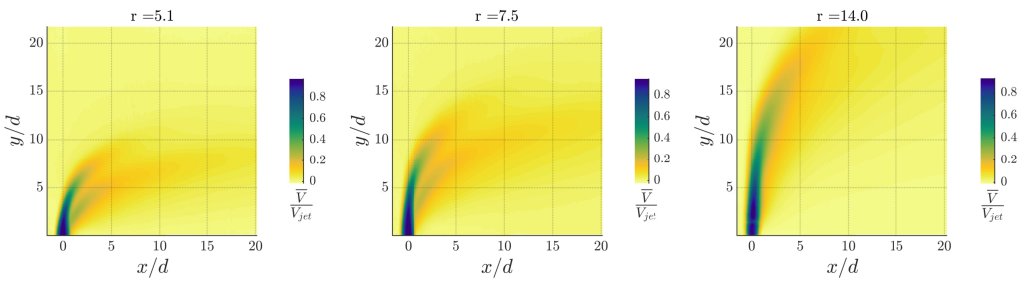

(b)

Figure 5.1: Contours of (a) the horizontal component of mean velocity normalized by jet exit velocity, and (b) the vertical component of mean velocity normalized by jet exit velocity of passive grid cases.

side of the jet in across all velocity ratios. This accelerated region is shown in the set of $\bar{u}$ profiles found in figure 5.3, at lowest velocity ratio considered, $r=3.8$ the acceleration is seen all the way to $10 d$ downstream of the jet exit. At the other extreme of velocity ratios, $r=14.0$ the acceleration behavior is much diminished, though can still be observed up to about $8 d$ from the jet exit. Beyond $8 d$ in figure 5.1a, the top edge of the jet extends to the top of the interrogation window, there is no view of the unaffected cross flow. The region of increased $\bar{u}$ is primarily a result of the jet bending as it corresponds with a region of diminishing magnitude in the $\bar{v}$, there is also a component of entrained crossflow which accelerates as it moves with the jet. Additionally, Andreopoulos and Rodi [19] observes acceleration on the windward side of the jet in cases where the jet exit velocity is less than that of the 

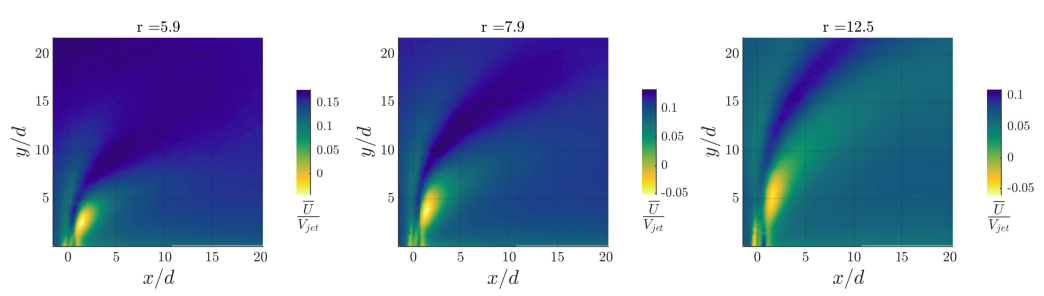

(a)
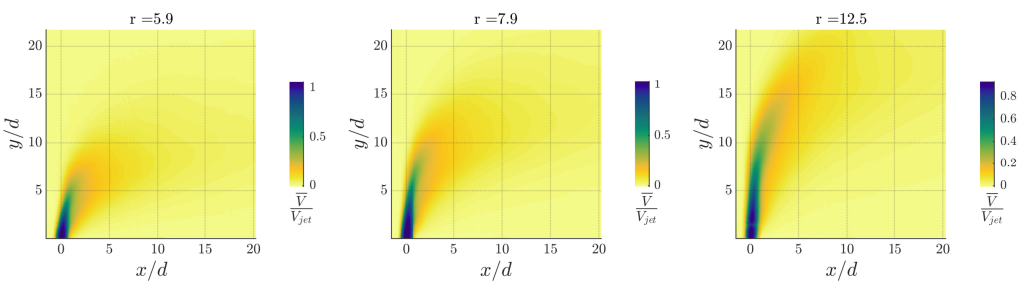

(b)

Figure 5.2: Contours of (a) the horizontal component of mean velocity normalized by jet exit velocity, and (b) the vertical component of mean velocity normalized by jet exit velocity of active grid cases.

cross flow concluding that the cross flow acts as a partial cover over the jet exit, causing bending at the exit resulting in $\bar{u}$ velocities in the jet fluid on the windward side greater than what is locally seen in the cross flow. At the high velocity ratio with weaker cross flows, the accelerated $\bar{u}$ very near the jet exit is not present, implying that in the lower velocity ratio cases, the windward sides acceleration is the result of the cross flow interaction.

The lee side of the jet shows negative $\bar{u}$ velocities which is consistent with recirculation behaviors seen in flows around a cylinder [85]. The jet flow is more complex than the often analogized cylinder example. A vortex system exists between the tunnel floor and the shear layer of the jet. Through comparisons between smoke seeding the jet, and smoke seeding of the cross flow, Fric and Roshko [20] find vortex structures 

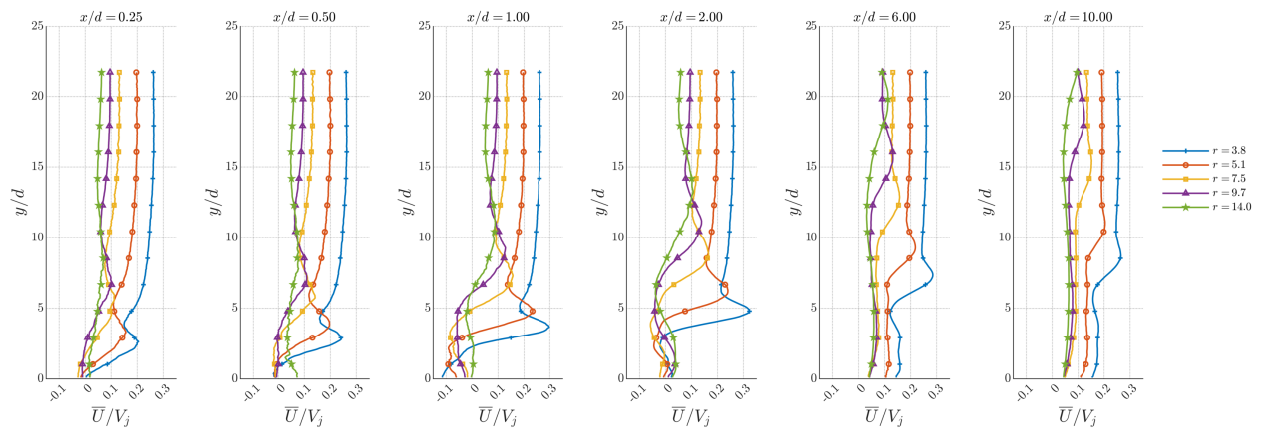

Figure 5.3: Passive grid profiles of the horizontal component of mean velocity.
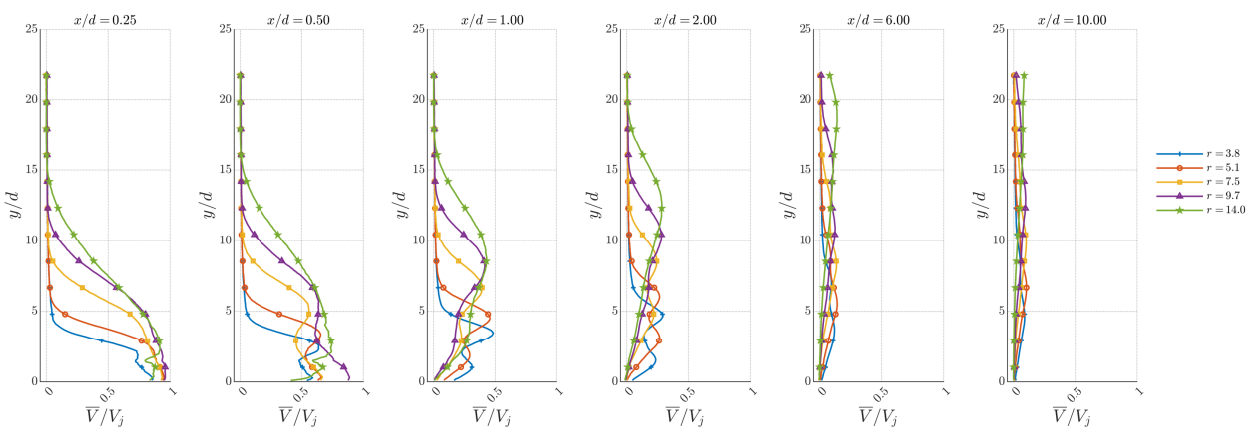

Figure 5.4: Passive grid profiles of the vertical component of mean velocity.

present in the wake of the jet originating in the cross flow boundary layer, a feature not present in the wake of a cylinder. The vortex system originating from the boundary layer shows the strongest behavior in the intermediate velocity ratios. The DNS conducted by Cortelezzi, Karagozian, et al. [86] shows significant changes direction in the crossflow fluid as it becomes entrained immediately to the lee side of the jet. These observations are consistent with the data of figures 5.1a and 5.3, the crossflow fluid changes direction as it closes around the core of the jet and becomes entrained in the jet flow. These effects are more pronounced at the lower velocity ratios as more defined recirculation regions occur at $r=5.1$ and $r=7.5$ and move away from the wall as $r$ increases. 

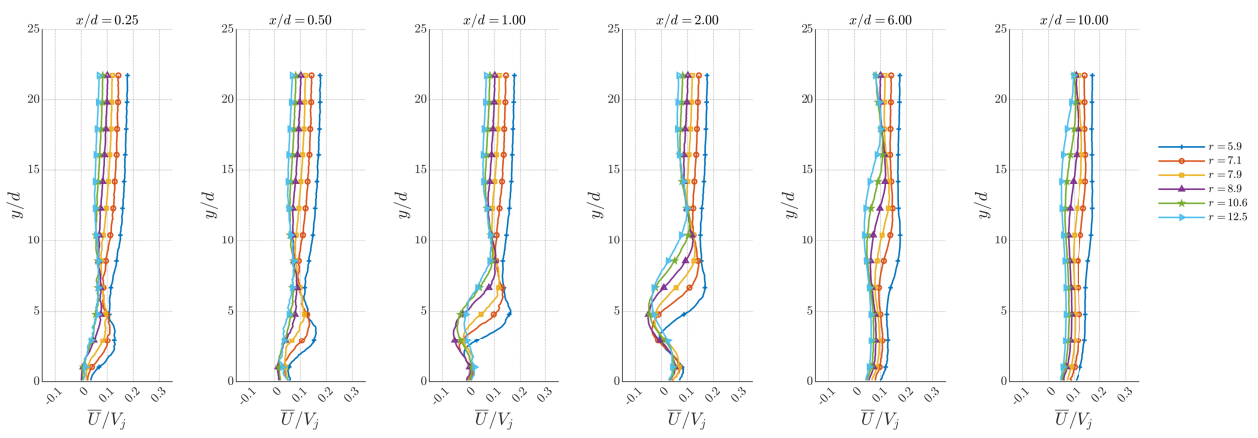

Figure 5.5: Active grid profiles of the horizontal component of mean velocity.
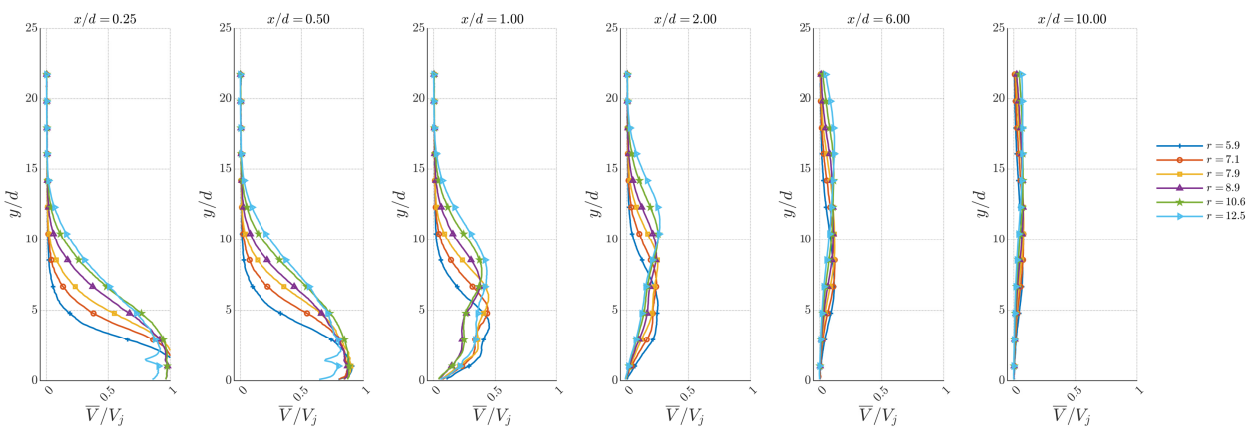

Figure 5.6: Active grid profiles of the vertical component of mean velocity.

The vertical component of velocity $\bar{v}$, shown for the passive grid cases in figure 5.1b. The jet core is the most prominent feature found in the vertical velocity field as this direction is aligned with the jet flow. Meaningful vertical velocities persist even after the core begins to bend. A region of increased vertical velocity is seen on the lee side of the jet core in the lower velocity ratios. This lee side behavior is consistent with entrainment processes described in [86]. Past experimental studies of turbulent jets into largely laminar crossflows show similar behavior in the $\bar{v}$ field [19] $[24]$.

Meaningful differences in the mean velocity fields between the passive and active grid sets are easily identified. The active grid mean velocities are shown as contours in 
figure 5.2 and profiles in figures 5.5 and 5.6 for horizontal $\bar{u}$, and vertical $\bar{v}$ velocities respectively. The region of accelerated flow on the windward side in the active grid set does not persist as far downstream as the same region in the passive grid cases. Similarly the lee side wake region of the active grid jets show $\bar{u}$ recirculation with less intensity and which diminishes closer to the jet exit. Contours of $\bar{v}$ show less bending of the potential core, this dissipation of the potential core tends to occur lower in the active grid cases as compared to passive grid cases. The area of separated increased vertical velocity in the wake region is not present in the active grid cases. The increased turbulence causes dramatic differences in the mean flow field. Much of the effects seen in the mean field could be explained by the turbulence borrowing energy from the mean field to maintain the more energetic fluctuations leading to the higher turbulence intensities. The general effect of the turbulence on the mean flow field is to diminish sharp differences. This is most clearly shown in the $\bar{v}$ profiles in figures 5.4 and 5.6. The active grid set shows a generally smooth profiles while the passive grid set has the double peak behavior.

\subsection{Centerlines}

The subsequent analysis requires an accurately defined centerline for each jet examined. Numerous attempts at defining universal behavior in centerline trajectories for the jet in cross flow can be found in the literature. Various scalings have been proposed to collapse centerlines to determine a universal behavior, Keffer and Baines [18] propose scaling by $r^{2} d$, though later Broadwell and Breidenthal [25] suggested that the global length scale in the flow is $r d$. The $r d$ scaling is the most 

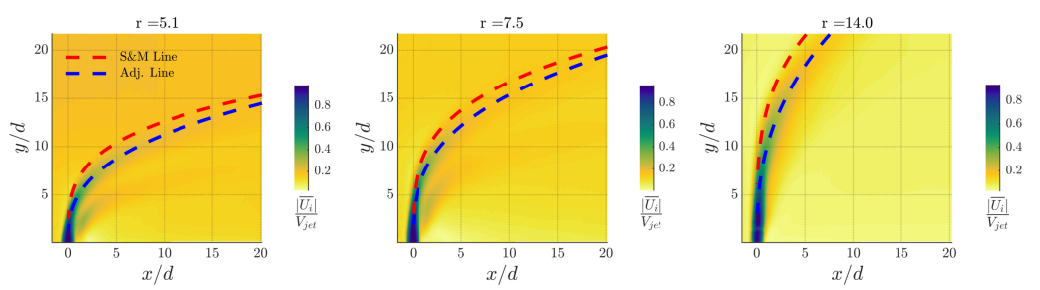

(a)
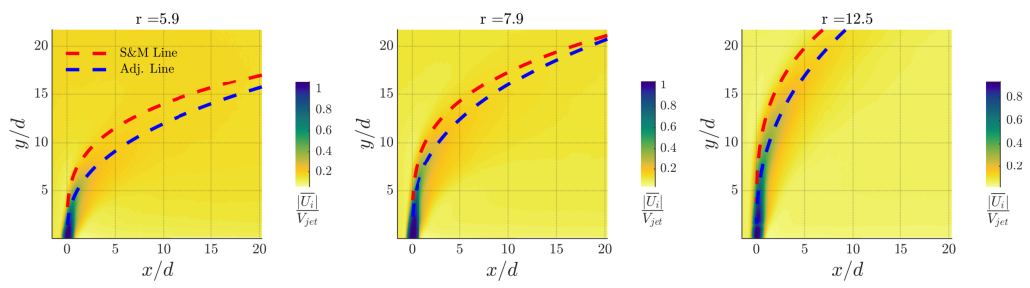

(b)

Figure 5.7: Centerlines from Smith and Mungal [21] and the improved centerlines plotted over contours of $\bar{v}$

commonly applied to achieve a centerline trajectory [87]. Smith and Mungal [21] compare various formulations and scalings and find that the simple power law relationship

$$
\frac{y}{r d}=A\left(\frac{x}{r d}\right)^{B}
$$

provides a reasonable approximation of the jet centerline trajectory based on the loci of velocity maxima with $A=2.05$ and $B=0.28$. Subsequent investigations have suggested an additional dependence on the boundary layer thickness of the crossflow [17]. 
Determining a more robust form for universal behavior in the trajectory is not a goal of this this work. To define the centerlines presently a data driven approach is employed. To this end, a simple algorithm is developed to define centerlines in the form of equation 5.1 with unique values for $A$ and $B$ for each jet. The centerline of a jet emanating into an ambient fluid is simply the center of the jet orifice, it is assumed the mean vertical velocity field forms a top-hat profile with the maximum velocity on the central axis of the jet [88]. In the presence of a crossflow, the component of mean velocity with a maximum at the jet centerline is the component locally tangent to the centerline as it rotates downstream of the jet exit. Maxima are determined based on a mean velocity field rotated about the local angle of the centerline $\theta$ with respect to the $\mathrm{x}$ axis by the rotation matrix

$$
\left[\overline{u_{r}}, \overline{v_{r}}, \overline{w_{r}}\right]=[\bar{u}, \bar{v}, \bar{w}]\left[\begin{array}{ccc}
\cos \theta & -\sin \theta & 0 \\
\sin \theta & \cos \theta & 0 \\
0 & 0 & 1
\end{array}\right]
$$

where $\left[\overline{u_{r}}, \overline{v_{r}}, \overline{w_{r}}\right]$ represent the mean velocity field rotated at each station in $x_{i}$ about $\theta_{i}$ as shown in figure 5.8. The initial rotation is preformed with a set of $\theta_{i}$ corresponding to centerline defined in (5.1) with the constants $A=2.05$ and $B=0.28$. A power law fit is performed on the points that locate the maxima of $V_{r}$, and the mean distance between the line which rotated the velocity field and the line representing the velocity maxima is computed. This process is repeated with centerline $n-1$ defining $\theta_{i}^{(n)}$, which in turn defines the rotated velocity field whose maxima form centerline $n$. The distance, $d_{c}$ is the length between centerline $n$ and 
$n-1$ measured on a line normal to centerline $n$ shown in figure 5.8. The rotation and maxima fitting process is repeated until the mean of $d_{c}$ at all $x_{i}$ falls below an arbitrary threshold, set at $0.01 d$.

Figure 5.7 shows the first centerline iteration, (5.1) with coefficients from Smith and Mungal [21] (S\&M line), and the last iteration after the mean distance threshold is met (Adj. Line). The S\&M line tends towards the windward side of the jet across the interrogated flow field while the Adj. line captures the true center of the jet, defined by the local maxima of velocity more accurately for both the passive grid and active grid cases.

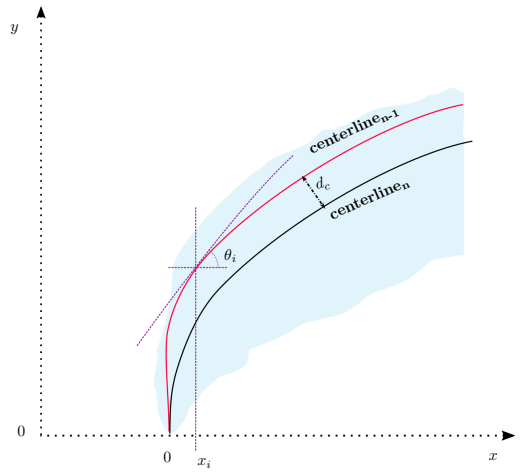

Figure 5.8: Schematic describing centerline refinement algorithm. Velocities are rotated about the set of $\theta_{i}$ based on centerline $n-1$, resulting rotated velocity maxima then define centerline $n$.

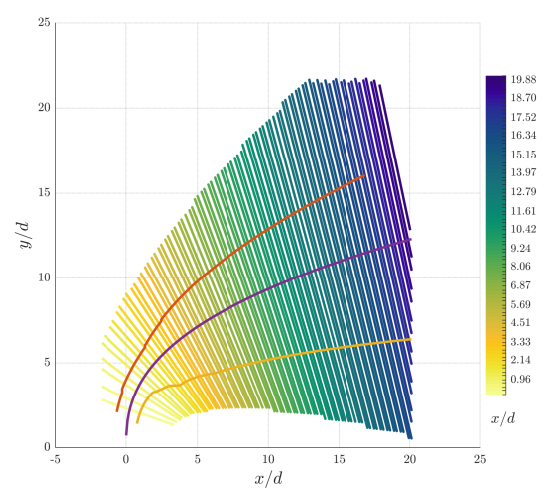

Figure 5.9: Locations of lines along which axial profiles are queried, each line is orthogonal to the centerline, colors correspond to the $x / d$ location at which the line intersects the centerline. Centerline and shear layers are shown to define the geometry of the jet. 

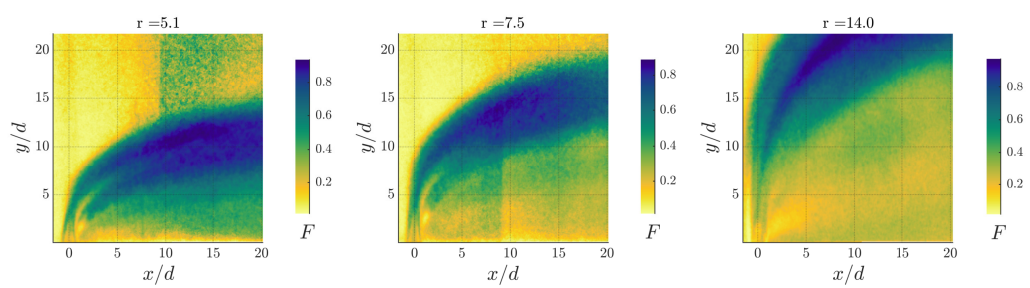

(a)
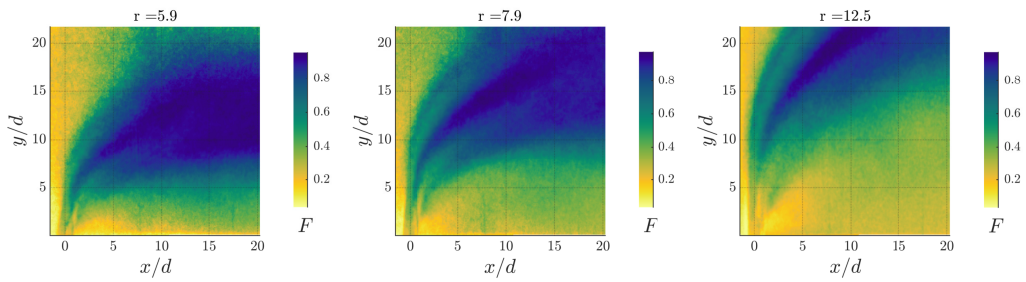

(b)

Figure 5.10: Contours of anisotropy parameter, $F$ for passive grid cases, (a) and active grid cases (b).

\subsection{Reynolds Stress Anisotropy}

The degree of isotropy in the Reynolds stress tensor can be examined through a single parameter $F$, defined in (3.9). Recalling what was briefly discussed in section 3, the function $F$ ranges from 0 to 1 representing states of purely two dimensional anisotropy and purely three dimensional isotropy, respectively. It should be noted, that $F$ does not provide information about the actual shape of the Reynolds stress tensor beyond the purely isotropic limit at a value of 1 .

Contours of $F$ across the interrogated flow field are shown in figure 5.10 for both the passive and active grid sets. Across all cases it is clear that the cross flow is strongly anisotropic. Minimum values of $F$ are seen in the passive grid $r=5.1$ and $r=7.5$ 
cases above the jet. A nearly isotropic core is seen across all cases, with development happening closer to the jet exit in the active grid set. The lower velocity ratio passive grid cases, $r=5.1$, and $r=7.5$ show a split in core of isotropy at values less than $\approx 5 x / d$. This area of elevated anisotropy within the more isotropic core occurs at a similar location to the recirculation region seen in $\bar{u} 5.1$. The effects of recirculation in the mean flow in the values of $F$ are less apparent in the higher velocity ratios. As the velocity ratio increases across the passive grid set, the extreme high values of $F$ become more localized to the center of the jet, and the edges of the jets core becomes less isotropic. In the active grid set, the bifurcation of the area of greater isotropy in the near field jet core is less apparent despite the presence of recirculation seen in figure 5.2a similar to the passive grid cases. The striking difference between the passive and active grid sets is the diffusion of the elevated degree of isotropy in the active grid cases suggesting that the turbulence of the cross flow is working to spread the effects of the jet across a wider area.

Radial profiles of $F$ are given in figure 5.11 and provide another view into the behavior as the flow progresses downstream. Profiles are along every line displayed in figure 5.9. The $x^{\prime} / d$ coordinate on the ordinate axis represents the distance along the profile with respect to the, with $x^{\prime} / d=0$ occurring on the centerline. The colors of the lines correspond to the distance downstream of the jet exit at which the profile crosses the centerline with values given by the color bars.

The profiles of $F$ show the differences between the passive and active grid crossflows more dramatically than the contours of figure 5.10. The active and passive grid sets have very different shapes to their profiles, the difference becomes more extreme as the flow develops downstream of the jet exit. Both sets achieve maximum values of 
$F$ at or near the centerline at approximately $10-12$ diameters downstream of the jet exit. Beyond this distance the decrease from the maximum value is small. On the windward side of the centerline, in the positive $x^{\prime} / d$, the passive grid set becomes much more anisotropic for all locations downstream. This tendency to become highly anisotropic is not seen as strongly in the active grid set. While the active grid cases show substantial decreases in $F$ at small distances downstream, as the flow progresses away from the exit, higher values of $F$ are maintained well above the centerline.

The examination of the invariant function $F$ reveals that the core of the jet is largely isotropic. This isotropy persists in the wake region, and is enhanced with downstream distance. The additional crossflow turbulence in the active grid set causes the isotropy of the core to be maintained well above the jet centerline. The elevated isotropy above the centerline is not seen in the passive grid set. In studies of a turbulent flow over a dunal bedform Dey et al. [89] show maximum values of $F$ in a boundary layer just above the region of separation and recirculation on the lee side of a dune, values of $F$ then decrease in the recirculation region. In the dune flow, the area just above the separated region will show a local extreme in mean velocity gradients. Measurements from a jet in crossflow with similar crossflow Re and a jet Re less than that considered here shows the rate of rotation and rate of strain tensors develop maxima near the centerline, and persist into the wake region [90]. The regions of increased velocity gradients correspond to areas of increased $F$ are seen in this work suggesting that the areas with more extremes in the gradients tend to be more isotropic. 

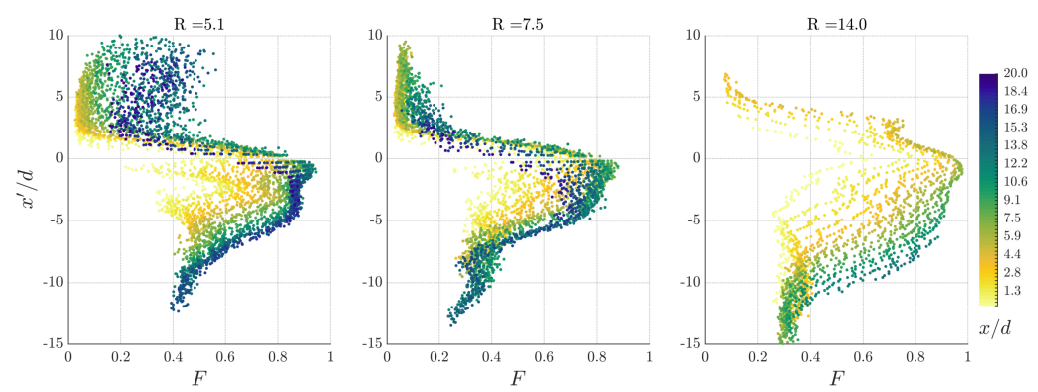

(a)
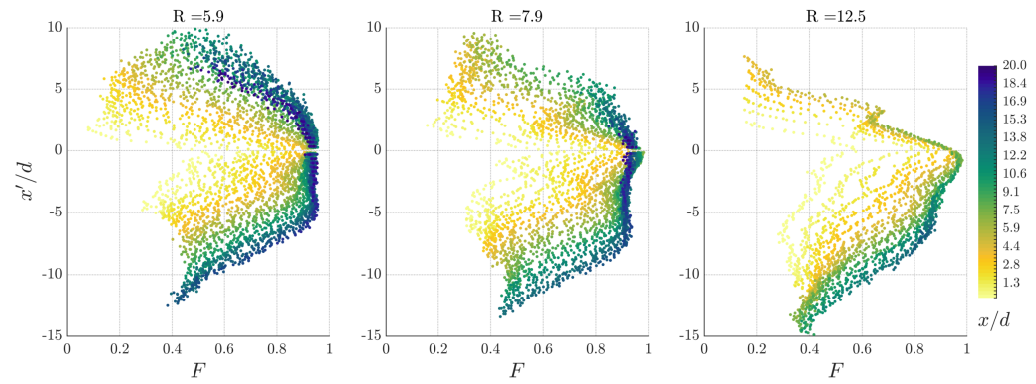

(b)

Figure 5.11: Profiles of anisotropy parameter, $F$ along lines orthogonal to the centerline. Colorbars represent the distance from jet exit at which the profiled line crosses the centerline. Passive grid cases, (a) and active grid cases (b).

Lumley triangles along discrete profiles taken along the a subset of lines orthogonal to the centerline displayed in figure 5.9 are found in figure 5.12. Each triangle is labeled according to the $x / d$ location at which the line intersects the centerline, different velocity ratios are indicated by the markers, and the distance along the line with respect to an origin at the centerline is represented by the color with all profiles sharing a common scale.

The Lumley triangles allow for the display of the real state of anisotropy, in contrast to the function $F$ which only tells how isotropic the turbulence is, but offers no information as to the shape of the anisotropy tensor. The Lumley triangles shown in figure 5.12 show that the anisotropy tensor is dominated by a single component along 

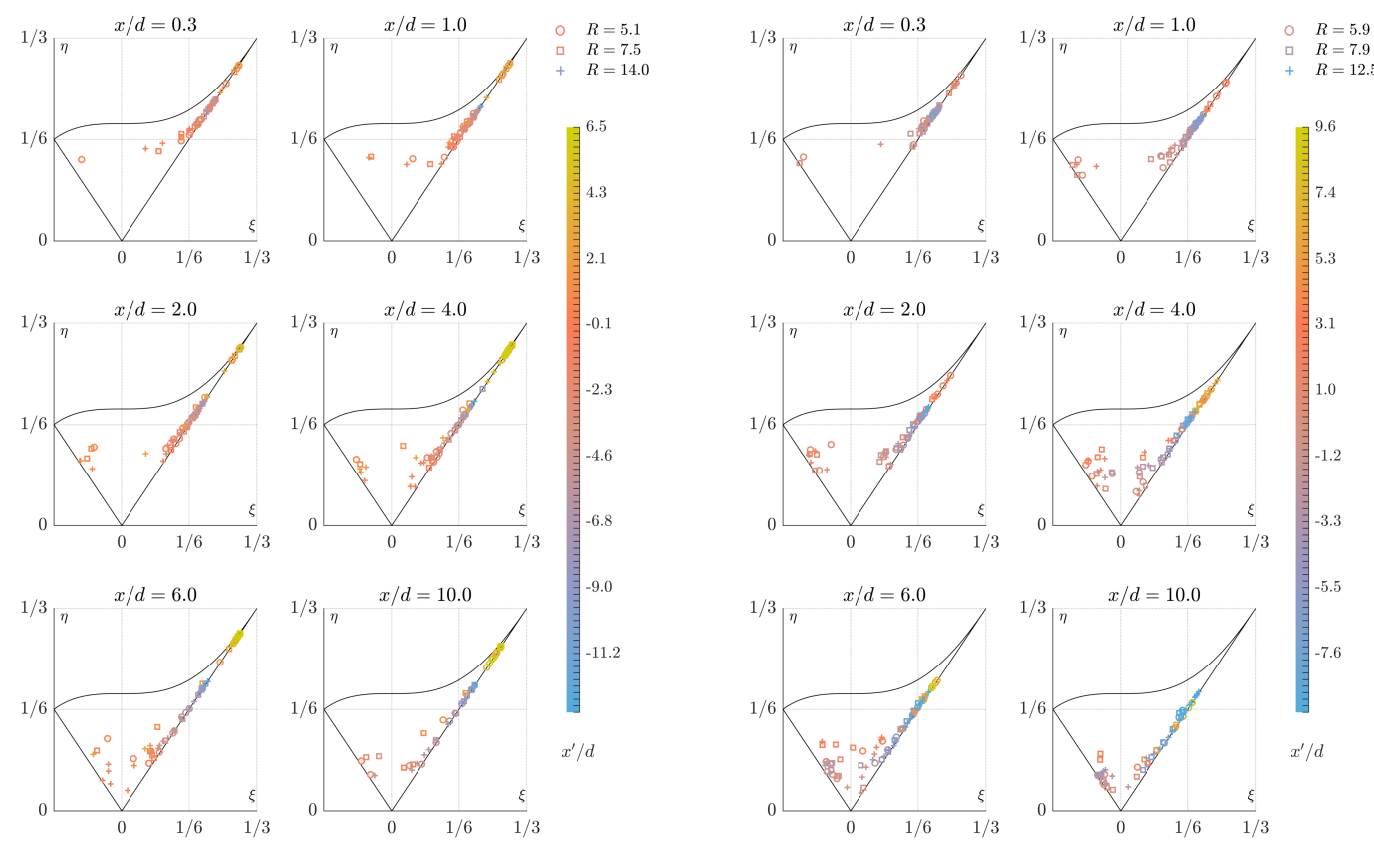

(a)

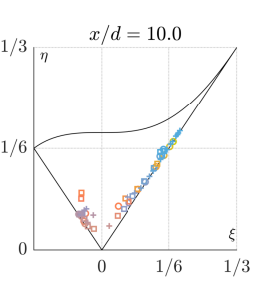

(b)

Figure 5.12: Anisotropy invariant maps (AIM), or Lumley triangles of six axial profiles about the jet centerline for the passive grid cases (a) and active grid cases (b).

most of the profiles close to the jet exit, $x / d \leq 1$. The majority of queried points in this region land on or near the positive $\xi$ axisymmetric line, this behavior is seen in both the active and passive grid sets and is the trend along all queried axial profiles. Observing the trend along the jet, both sets of cases show high $\xi$ approaching the one component limit near the jet exit, though in the active grid cases the largest $\xi$ values fall away from the limit along the length of the jet. The maximum value of $\xi$ in the passive grid set is 0.3 at the extreme positive end of the profile at $x / d=4$, while the maximum $\xi$ in the active grid cases occurs again above the jet but along the $x / d=0.25$ profile. As observed in figure 5.11, the profiles of $F$, the cross flow is shown to be the least isotropic portion of the flow, with values of $F$ falling sharply towards zero above the jet centerline in the passive grid set, and more slowly in the active 
grid set. This observation is supported by the Lumley triangles in figure 5.12, queried points that are farthest above the centerlines, depicted as the most yellow, tend to fall further up the axisymmetric line towards the one-component limit particularly in the passive grid cases. The region where the Reynolds stress tensor shows greater isotropy falls towards the center of the jet. The "strength" of the isotropy tends to be larger as distance from the jet exit is increased, and the region where the greater degree of isotropy is seen spreads radially about the jet centerline, just as the jet width grows downstream. This relative tendency towards isotropy about the jet core is more apparent in the active grid, where there is a greater area of both negative $\xi$, and smaller $\eta$. The larger area of the active grid profiles which falls near the isotropic limit is consistent with the observations of $F$ larger isotropic core seen in both the profiles and contours of $F$.

A common feature of the Lumley triangles across all cases considered is a tendency for the windward extreme of the profiles to approach the one component limit. This indicates that the strain rate tensor along the windward edge of the shear layer is dominated by a single shearing component. Single component strain rate tensors drive the Reynolds stresses to a one component limit. Initially isotropic homogeneous turbulence introduced to a free shear flow with a strain rate $S_{i j}=S_{12}$ will, over infinite time achieve an anisotropy tensor with only a $b_{11}$ non zero component [91]. Single component strain rate tensors are a feature of the turbulent boundary layer and have been found to be the primary driver of streaky structures the breakup of which play a dominant role in turbulence production in the boundary layer [92]. The anisotropy along the shear layer leads to enhanced turbulence production which itself will lead to a further anisotropic state [93]. The contours of the mean flow fields in the active grid cases of figure 5.2 show decreased horizontal acceleration along the 
windward edge of the jet and a more diffused core of vertical velocity as compared to those of the passive grid cases in figure 5.1. The differences in the mean flow fields suggest that there are larger mean gradients along the windward side of the jet in the passive grid set. The smaller mean gradients in the active grid set could be a driving mechanism for the ability of the more isotropic core to persist across the windward shear layer.

Figures 5.13 and 5.14 show the relative magnitudes of the components of the anisotropy tensor $a_{i j}$ given in (2.15). Profiles are again taken along a subset of axial profiles shown in figure 5.9, the origin is the jet centerline. Plots are labeled with respect to the $x / d$ coordinate at which the profile intersects the centerline. The background colors correspond to the colors in the zone definitions found in figure 5.15 .

Normalization of the profiles in figures 5.13 and 5.14 is carried out differently for the diagonal contributions from the normal stresses and the off diagonal shear stress components as the shear stresses are about an order of magnitude smaller than the normal stresses. Each set of stresses is locally normalized to the component of greatest magnitude along the profile. This normalization allows for the stresses to be evaluated as they evolve together along the profiles, but does not allow for a direct comparison between values of different profiles. Values of $F$ are also reported in figures 5.13 and 5.14 which allows for an assessment of the evolution of $F$ with respect to the evolution of $a_{i j}$.

In the passive grid $r=5.1$ and $r=7.5$ cases in figure 5.13 show that in Z1, which is represents the crossflow, $a_{i j}$ is dominated by the out of plane $\overline{w^{\prime} w_{a}^{\prime}}$. Along the 
$x / d=1$ profile the large $\overline{w^{\prime} w_{a}^{\prime}}$ values develop just beyond the lee side shear layer. Peak values occur near the centerline, then inflect at about the windward shear layer and finally gain magnitude as the profile continues into the crossflow. The $\overline{w^{\prime} w_{a}^{\prime}}$ component within $r=14$ case follows a similar profile within the jet, though the magnitude does not recover at the windward shear layer. Along the same $x / d=1$ profile, considering the full balance of the normal components, in the the $r=5.1$ and $r=7.5$ cases. Through Z4, the wake region outside of the lee side shear layer the normal components are all smaller than seen in the crossflow. This indicates that the fluctuations are modulated in the direct wake of the jet. The moderate values of $F$ seen in these intermediate velocity ratio cases show that this region of the flow has an approximate balance in $a_{i j}$ leading to a greater level of isotropy. A similar conclusion is difficult to make with respect to the $r=14$ case along the same profile as the flow geometry does not allow for as much of the crossflow to be seen in the data. Though the failure of $F$ to recover magnitude after the centerline peak suggests the normal components are in a less balanced state within the crossflow than in the wake.

Within the jet it is helpful to examine the profile of $F$ and consider the balance of stresses. Still along the $x / d=1$ profile in the $r=5.1$ case, the profile of $F$ has 2 peaks. One peak in $F$ occurs on the lee side of the shear layer, and the other near the centerline. The first peak corresponds to an increase in $\overline{w^{\prime} w_{a}^{\prime}}$ just to the lee side of the shear layer. The peak corresponds to an area where $\overline{w^{\prime} w_{a}^{\prime}}$ begins to increase ahead of the increases in magnitude of $\overline{u^{\prime} u_{a}^{\prime}}$ and $\overline{v^{\prime} v_{a}^{\prime}}$. The second peak occurs as $\overline{u^{\prime} u_{a}^{\prime}}$ crosses $\overline{v^{\prime} v_{a}^{\prime}}$ at the centerline. 
The other two cases, $r=7.5$ and $r=14$ do not show the double peak in $F$ along the $x / d=1$ profile. In the higher velocity ratios $F$ increases until the lower half of the jet in Z3, this corresponds roughly to the peak in $\overline{v^{\prime} v_{a}^{\prime}}$. Beyond this point the stresses continue unbalanced as they alternately swing in magnitude through Z2. Past the windward shear layer all the lower $r$ cases the 3 normal stress components increase in magnitude. These increases are unbalanced as the rate of increase in $\overline{w^{\prime} w_{a}^{\prime}}$ is much greater than the rates of $\overline{u^{\prime} u_{a}^{\prime}}$ and $\overline{v^{\prime} v_{a}^{\prime}}$ which are nearly identical to the extents of the profile. In $r=14$ the 3 normal components tend back towards zero in Z1.

The $\overline{u^{\prime} v_{a}^{\prime}}$ component along $x / d=1$ tends to follow a common pattern across the three velocity ratios. Values of $\overline{u^{\prime} v_{a}^{\prime}}$ swing from positive to negative within the jet, this swing is centered on the centerline. The $\overline{u^{\prime} w_{a}^{\prime}}$ component spikes along Z3 and falls off quickly at the centerline. In the lower velocity ratio cases $\overline{u^{\prime} w_{a}^{\prime}}$ tends to go negative with increasing magnitude into the crossflow, while at $r=14$ it goes to zero with the other components and $F$. The shear component $\overline{v^{\prime} w_{a}^{\prime}}$ similarly tends to go positive, lagging $\overline{u^{\prime} v_{a}^{\prime}}$, then falling back down and fluctuating near zero in Z1.

The trends seen in the $x / d=1$ profile across the passive grid cases reveal a generally equal distribution of the components of $a_{i j}$ to the lee side of the jet indicated by elevated values of $F$. As the windward shear layer is approached the out of plane $\overline{w^{\prime} w_{a}^{\prime}}$ increases in magnitude correlating to a spike in $F$. Within the jet the other two normal components typically go negative in Z3, followed by negative shearing stresses in Z4. In the crossflow, the two lower velocity ratios show the $\overline{u^{\prime} u_{a}^{\prime}}$ and $\overline{v^{\prime} v_{a}^{\prime}}$ components become more negative along with $\overline{u^{\prime} w_{a}^{\prime}}$. This occurs while the $\overline{w^{\prime} w_{a}^{\prime}}$ 

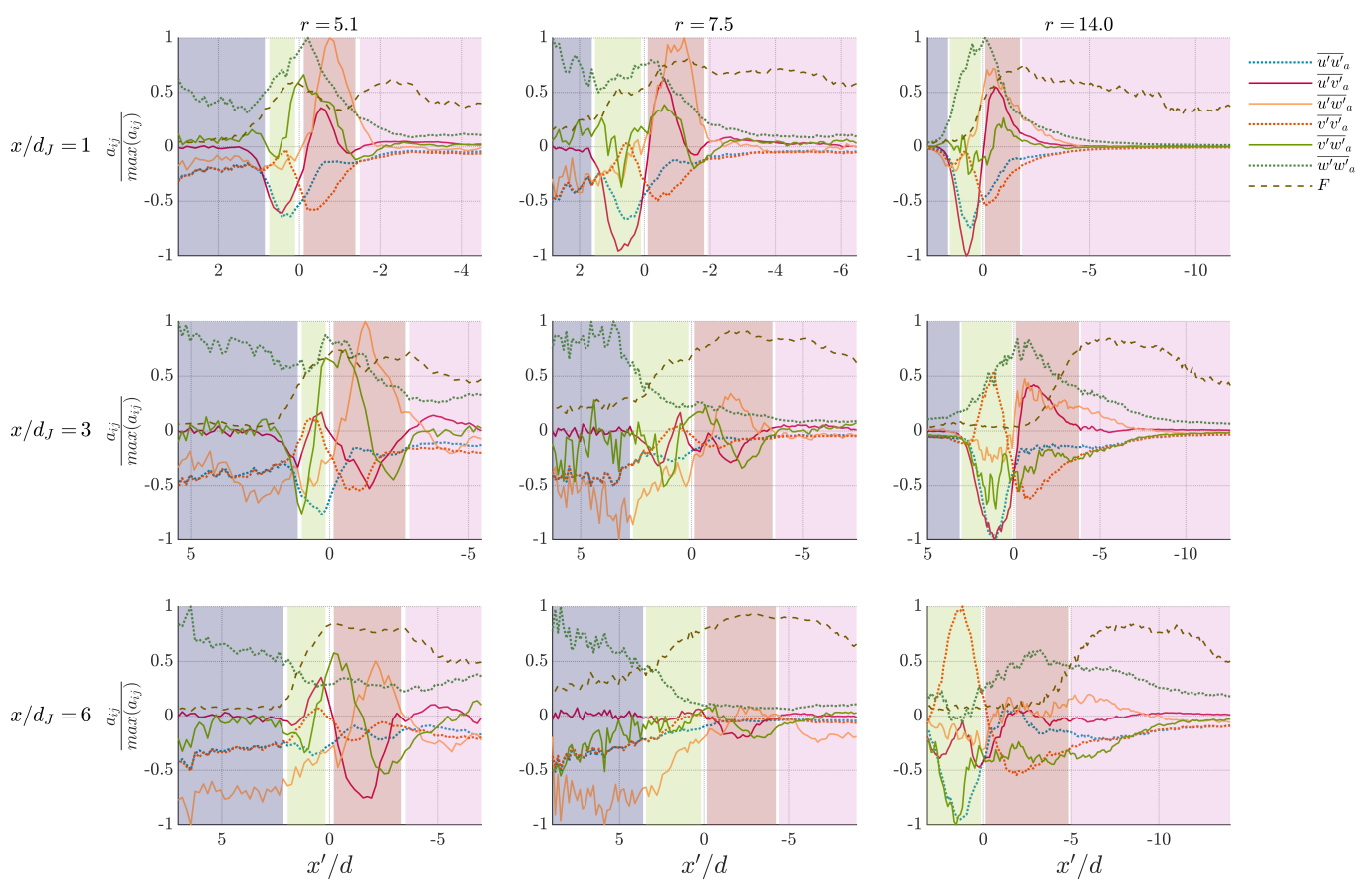

Figure 5.13: Components of the passive grid cases anisotropy tensor $a_{i j}$ defined in equation (2.15). Diagonal tensor components normalized by the local maximum of the diagonal, and the off diagonal components similarly normalized by the maximum of the off diagonal component along each queried profile.

component increased at a much greater rate. The other components tend towards zero in in the crossflow. The $r=14$ case shows different behavior in $\mathrm{Z} 1$ where all the values go towards zero along with $F$. The trend towards zero in Z1 differs from the distribution of components in $\mathrm{Z} 4$, as in the wake region the components are more balanced as $F$ is elevated.

Along the $x / d=3$ profiles in the passive grid cases similar patterns appear in the profiles of $F$. The double peak in $F$ is again seen in only $r=5.1$ due to the increased activity in $\overline{v^{\prime} v_{a}^{\prime}}$ in Z3. Additionally the spread seen between the 3 normal components in $r=5.1 \mathrm{Z} 4$ is much wider, while their balance is roughly the same as seen in this region on the $x / d=1$ profile. The large negative peak in $\overline{v^{\prime} v_{a}^{\prime}}$ in $\mathrm{Z} 3$ is not seen in 
the $x / d=3$ profile of $r=7.5$ as it was in the $x / d=1$ profile, $\overline{u^{\prime} u_{a}^{\prime}}$ and $\overline{v^{\prime} v_{a}^{\prime}}$ appear to oscillate about each other within the jet.

The distribution in components in the $r=14$ case acts quite differently. In this higher velocity ratio $F$ peaks as $\overline{w^{\prime} w_{a}^{\prime}}$ begins to increase in $\mathrm{Z} 4$. The balance in components leading to the peak in $F$ is lost to the lee side of the upper shear layer. There the increase in $\overline{w^{\prime} w_{a}^{\prime}}$ outpaces the increase in $\overline{v^{\prime} v_{a}^{\prime}}$ which reaches a maximum magnitude in Z2. A very large increase in the $\overline{u^{\prime} u_{a}^{\prime}}$ component is seen in Z2, driving $F$ practically to zero. $F$ remains near zero as the magnitude of $\overline{w^{\prime} w_{a}^{\prime}}$ remains elevated while the other two normal components go to zero.

The $\overline{u^{\prime} v_{a}^{\prime}}$ shear component along $x / d=3$ in the $r=5.1$ case switches sign within the jet compared to the $x / d=1$ station. In the profile at $x / d=1 \overline{u^{\prime} v_{a}^{\prime}}$ is positive in Z3, and negative in Z2, while at $x / d=3$, it is negative in Z3 and positive in Z4. The same component along in the 7.5 case fluctuates across the jet rather than maintaining the swing seen across all of the $x^{\prime} / d=1$ cases. In the $r=14$ case this behavior in $\overline{u^{\prime} v_{a}^{\prime}}$ returns. The other two shear stresses act largely the same as described in the $r=5.1$ case for both $x / d=1$ and $x / d=3$. Similarities across the two stations are also seen in $r=7.5$ and $r=14$ with the shearing stresses being smaller in magnitude.

The $x / d=6$ profile of $r=5.1$ shows the double peak in $F$ becoming less pronounced as the behavior of $\overline{u^{\prime} u_{a}^{\prime}}$ and $\overline{v^{\prime} v_{a}^{\prime}}$ change. Instead of an oscillation about the centerline, $\overline{u^{\prime} u_{a}^{\prime}}$ and $\overline{v^{\prime} v_{a}^{\prime}}$ oscillate about each other twice within Z3, but at smaller magnitudes. In $\mathrm{Z} 2, \overline{w^{\prime} w_{a}^{\prime}}$ increases more slowly compared to the other stations. On the lee side of the centerline in $\mathrm{Z} 3 \overline{w^{\prime} w_{a}^{\prime}}$ begins to increase more rapidly which drives $F$ lower. In 
Z1 $\overline{u u_{a}}$ and $\overline{v v_{a}}$ move together and diverge from $\overline{w w_{a}}$ as the out of plane component rapidly increases magnitude. The $r=7.5$ case on the same profile shows the stress profile of the previous stations beginning to breakdown. The large swing of $\overline{u^{\prime} v_{a}^{\prime}}$ from positive to negative across Z1 and Z2 is no longer apparent. The normal stresses show little activity until past the centerline where $\overline{w^{\prime} w_{a}^{\prime}}$ begins to diverge from $\overline{u^{\prime} u_{a}^{\prime}}$ and $\overline{v^{\prime} v_{a}^{\prime}}$ moving together. The relative balance in components that had existed to the lee side of the centerline is lost as the profile traverses to the windward side. At $x^{\prime} / d=6$ the $r=14$ case also begins begins to show breakdown of its earlier patterns. The $\overline{u^{\prime} v_{a}^{\prime}}$ no longer shows a strong positive values in Z3 but instead only becomes negative before the centerline. Increases in $\overline{w^{\prime} w_{a}^{\prime}}$ in $\mathrm{Z} 4$ combined with a divergence between $\overline{u^{\prime} u_{a}^{\prime}}$ and $\overline{v^{\prime} v_{a}^{\prime}}$ drive the values of $F$ to near zero just inside the jet. A balance in components is never recovered as the profile traverses towards the windward side. Due to the flow geometry, the crossflow is not accessible along the $x / d=6$ profile in the $r=14$ case.

Comparing the observations on the passive grid cases to the active grid set similar behaviors are seen along the profile at $x / d=1$ up to the windward side of the jet. In Z4 there are elevated values of $F$ while the normal stresses cluster around zero with little variation. As the lee side shear layer is approached the normal components begin to diverge. The $\overline{w^{\prime} w_{a}^{\prime}}$ component becomes more positive as $\overline{u^{\prime} u_{a}^{\prime}}$ and $\overline{v^{\prime} v_{a}^{\prime}}$ become more negative. On the windward side of the jet the balance begins to breakdown and $F$ goes towards zero. In the $\mathrm{Z} 1$ region of the $r=5.9$ case there is divergence between $\overline{w^{\prime} w_{a}^{\prime}}$ and the other two normal components $\overline{u^{\prime} u_{a}^{\prime}}$ and $\overline{v^{\prime} v_{a}^{\prime}}$, which evolve together. This divergence is accompanied by near zero values in $F$. The higher velocity ratio cases all have normal components approaching zero along with $F$ into the crossflow, a similar configuration as the $r=14$ case in the passive grid set. 

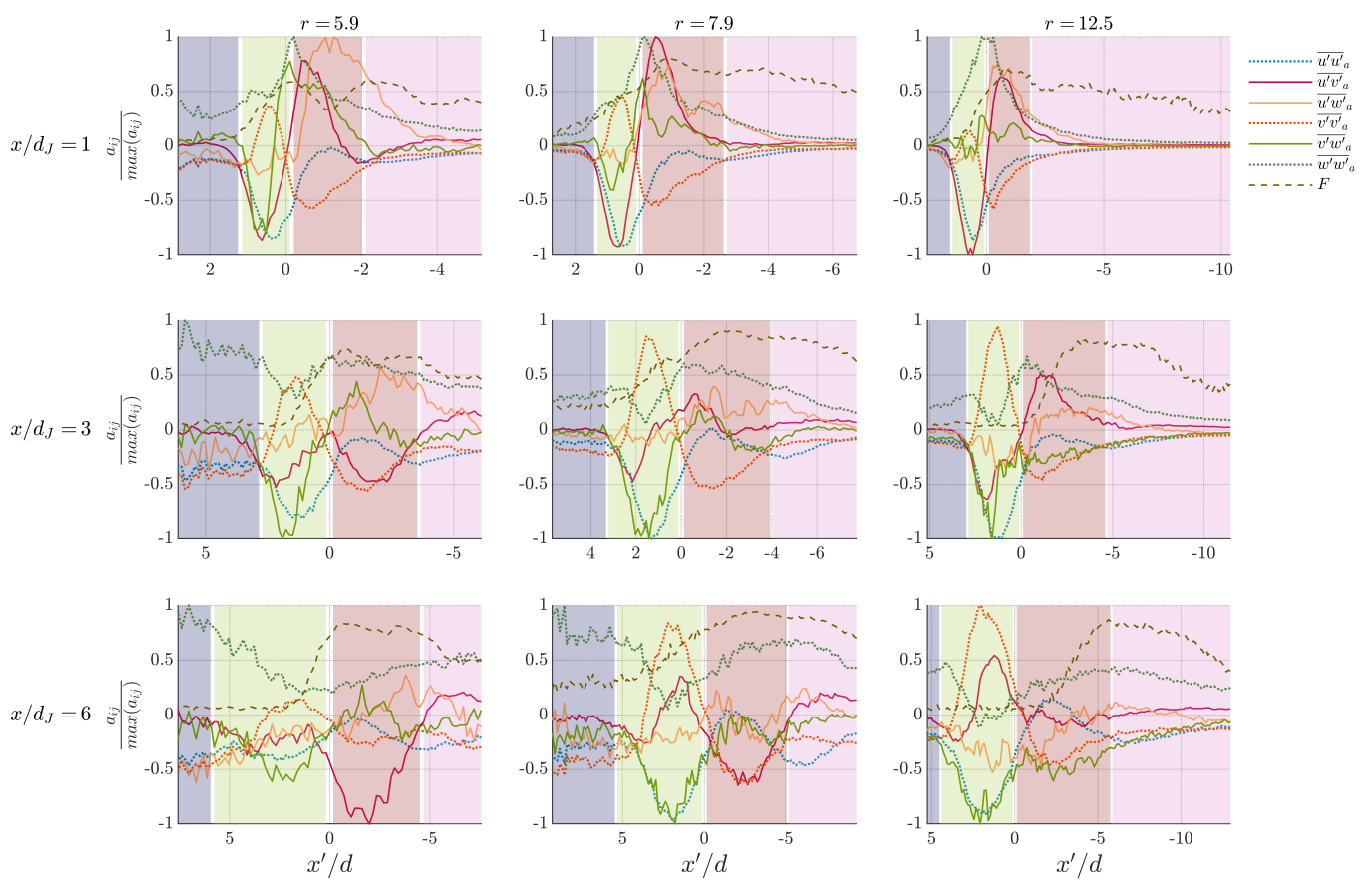

Figure 5.14: Components of the active grid cases anisotropy tensor $a_{i j}$ defined in equation (2.15). Diagonal tensor components normalized by the local maximum of the diagonal, and the off diagonal components similarly normalized by the maximum of the off diagonal component along each queried profile.

The set of profiles for the active grid cases largely mimics what is seen in the passive grid. The $\overline{u^{\prime} v_{a}^{\prime}}$ component changes sign as the 5.9 case goes from $x / d=1$ to $x / d=6, F$ tends to drop sooner than the other cases in the $r=12.5$ case, etc. The intermediate velocity ratio shows $F$ maintaining a positive value to the end of the profile in both the passive and active grid sets despite substantially different distributions of components. In the active grid $r=7.9$ case at both $x / d=3$ and $x / d=6$ the $\overline{v^{\prime} w_{a}^{\prime}}$ component tracks closely with the $\overline{u^{\prime} u_{a}^{\prime}}$ from Z3 out to the edge of the jet. This correlation between $\overline{v^{\prime} w_{a}^{\prime}}$ and $\overline{u^{\prime} u_{a}^{\prime}}$ appears in the passive grid $r=7.5$ case at $x / d=6$ as well, though the correlation is not as strong. This correlation does not 
appear in the $r=3$ case, though there is activity in $\overline{v^{\prime} w_{a}^{\prime}}$ which is not seen in the other profiles.

The profiles of the anisotropy tensor components in figures 5.13 and 5.14 suggest that the tendency towards the one component limit in the windward side of the jet as seen in the Lumley triangles of figure 5.12 appears to be driven by the out of plane fluctuations. This is evident by the elevated $\overline{w^{\prime} w_{a}^{\prime}}$ component on the windward side of the centerlines in both the active and passive grid sets, increased activation in $\overline{u^{\prime} w_{a}^{\prime}}$ and $\overline{v^{\prime} w_{a}^{\prime}}$ are also seen. The windward side of the jet sees the initial interaction with the crossflow. The crossflow tends to deflect around the windward side of the jet rather than be directly entrained, much of the entrainment of crossflow fluid into the structures of the jet occurs on the lee side [86]. The deflection will result in energy from the mean flow changing direction, with some of this change going to the out of plane motions, large mean gradients will be associated with this activity. In addition to the deflection of the mean flow, the counter rotating vortex pair brings with it substantial out of plane fluctuations in the velocity field [32]. Shear layer vortices provide additional dynamics to the windward edge of the jet and are relatively energetic. Meyer et al. [94] through proper orthogonal decomposition of the mean velocity field find that the shear layer vortices contain about six percent of the total energy contained in the flow, through instantaneous velocity snapshots the shear layer vortices are shown to contribute substantially to the fluctuating field. The profiles show increased activation in the in plane components at station $x / d=6$ which is roughly the area where the POD analysis shows their development [94]. As the components of $a_{i j}$ are generally less stable in the active grid set, the crossflow turbulence could be playing a role in redistributing some of the energy between the components and dampening the dominance of the out of plane component. 


\subsection{Principle Axes Rotation}

As described in section 3.1.1, the eigenmatrix of the normalized anisotropy tensor, defined in (3.5) and (3.6) form the set of principle axes of $b_{i j}$. The principle axis system can then be represented by a four dimensional quaternion, which in a three dimensional sense defines the vector $\mathbf{n}$ and the angle $\theta$. In any state of anisotropy the principle axes of $b_{i j}$ are rotated $2 \theta$ about $\mathbf{n}$ with respect to the global coordinate system. An examination of these parameters could allow for a better understanding of the evolution of the anisotropy tensor throughout the flow.

In order to depict the axis of rotation tensor, $\mathbf{n}$ an octant analysis scheme is devised to define the residence octant of $\mathbf{n}$. The octant analysis scheme is inspired by conditional averaging techniques developed to filter Reynolds shear stress signals. In the context of Reynolds stresses, component directions are based on the relative strengths of the fluctuating velocity components. Two dimensional quadrant analysis has become a widely used technique to identify bursts and sweeps in flows containing turbulent

Table 5.1: Residence octant of $\mathbf{n}$ based on the sign of its components.

\begin{tabular}{cccc}
\hline Octant & $n_{x}$ & $n_{y}$ & $n_{z}$ \\
\hline 1 & + & + & + \\
2 & + & + & - \\
3 & - & + & - \\
4 & - & + & + \\
5 & + & - & + \\
6 & + & - & - \\
7 & - & - & - \\
8 & - & - & + \\
\hline
\end{tabular}




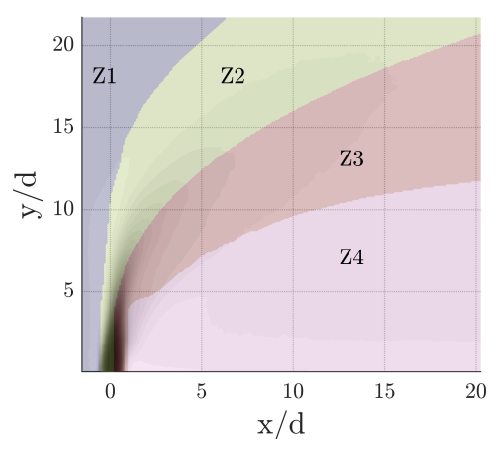

Figure 5.15: The flow broken up into 4 zones based on the shear layer and centerline, data shown under color map for example is $\left|\bar{u}_{i j}\right|$ of active grid $r=7.9$.

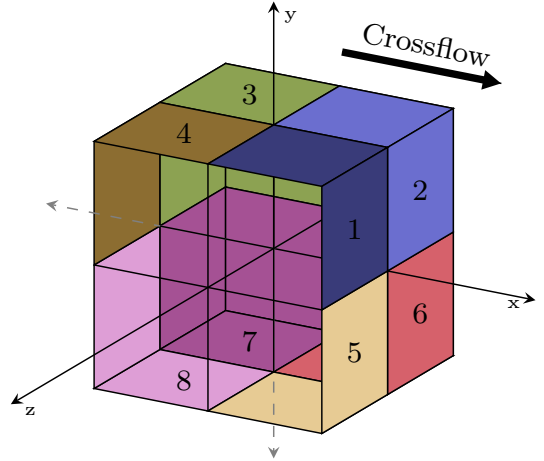

Figure 5.16: Octant definitions in which the residence of the rotation vector is defined.

boundary layers [95] [96]. When data are in three dimensions, quadrant analysis is extended to octant analysis. In the context of classifying boundary layer ejection events octants are defined in a Cartesian coordinate system, and events are classified according to the direction of the fluctuating velocity vector $u_{i}^{\prime}$ [97]. In the same manner, octants are defined across the coordinate system of the jet with the first octant defines $x, y$, and $z$ as all positive. Moving counter clockwise in the $z x$ plane three more octants are defined with a positive $y$ component. The same process is repeated for the negative $y$ portion of the coordinate system. Figure 5.16 shows a diagram of the octant definition, and the octant binning logic is displayed in table 5.1 .

The residence octants of $\mathbf{n}$ across the entire interrogated flow field for the six cases considered are shown in figure 5.17 with a centerline for reference. The passive grid $r=5.1$ and $r=7.5$ cases show little effect in the region above the centerline, the inflow conditions seem to persist until just above the centerline. This behavior is impossible to assess for the passive grid $r=14.0$ case due to the flow geometry 
as the jet flow goes beyond the upper extents of the data with little view of the crossflow. Comparing the low $r$ passive grid cases to the $r=5.9$ and $r=7.9$ cases, substantially more activity in $\mathbf{n}$ is seen above the centerline in the active grid cases.

It has been observed that the center of the counter rotating vortex pair follows a trajectory below that of the centerline [98]. Additionally, the counter rotating vortex pair has been shown in simulations to entrain the crossflow on the lee side of the jet and transport it up [86]. As the active grid set is in a higher turbulence intensity regime, the crossflow carries more energy in the turbulence. This could account for the observed increase in the rotation of $\mathbf{n}$ on the windward side of the jet centerline. The energy laden crossflow is pushed up through the jet causing the dynamics seen
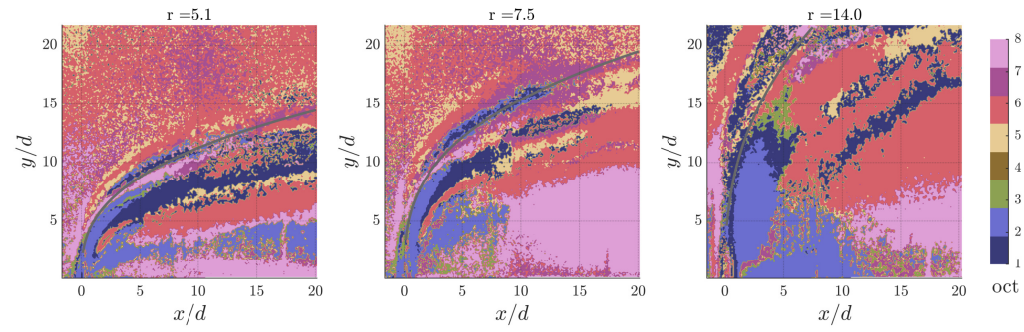

(a)
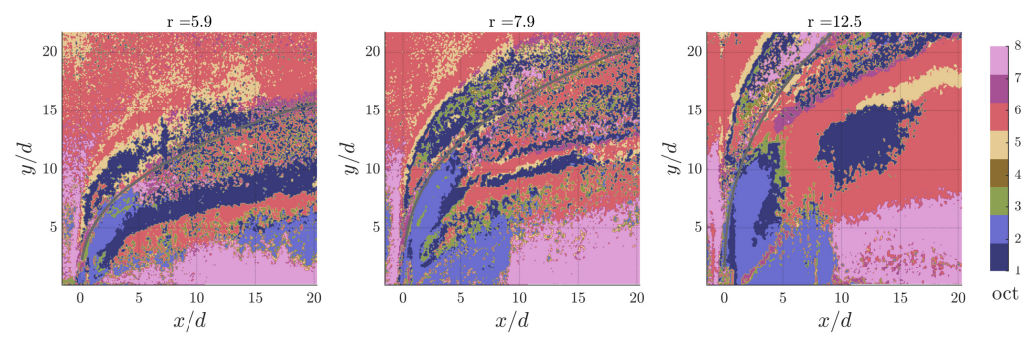

(b)

Figure 5.17: Residence octants of the axis of rotation, $\mathbf{n}$ as described in (3.13). Passive grid cases (a) and active grid cases (b) 

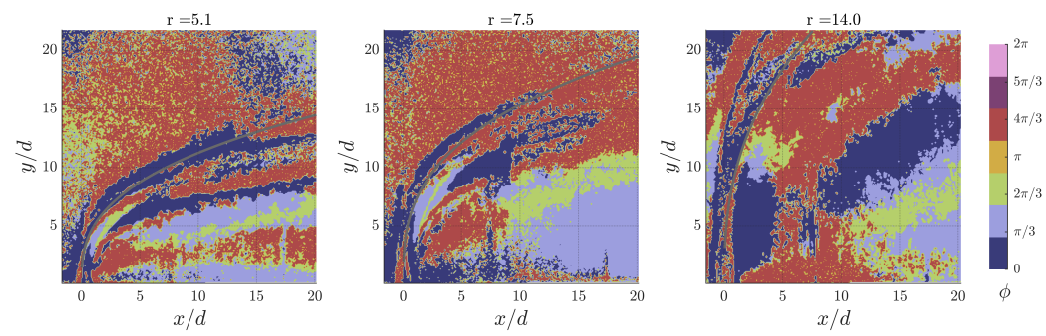

(a)
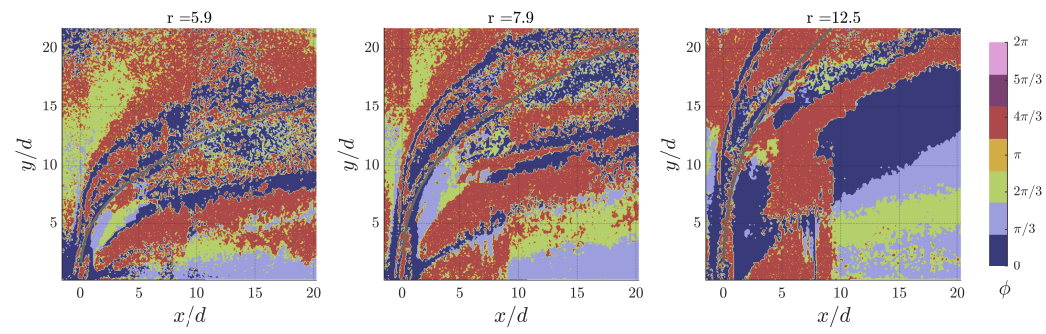

(b)

Figure 5.18: Angle of rotation $\theta$ about $\mathbf{n}$, as described in (3.13). Passive grid cases (a) and active grid cases (b)

by the individual components of the fluctuating velocities to change directions. This change in the fluctuations leads directly to a change in the state of the Reynolds stress anisotropy. In the active grid set this increased rotation in the principle axes can be seen across a larger area of the windward side of the jet than in the less energetic passive grid crossflow conditions.

Figure 5.18 shows the angle of rotation about $\mathbf{n}$. A consequence of the mapping from the 4 dimensional quaternion space to the 3 dimensional rotation space is a double mapping of the rotation space, $\underline{\mathbf{q}}=-\underline{\mathbf{q}}$. Because of the double mapping the angle defined directly via $\underline{\mathbf{q}}$ is confined to $0 \leq \theta \leq \pi$ but encodes the complete 3 dimensional rotation space. Here the angle $\phi$ is introduced, defined over the entire rotation domain of $0 \leq \phi \leq 2 \pi$, where $\phi=2 \theta$. The quaternion derived angle, $\phi$ can 
describe a complete rotation of the principle axes from 0 to $2 \pi$. Due to the sensitivity of this parameter to fluctuations in in the flow, and the compounding effects of error in the multi step processing, the resulting parameter contains a great deal of noise. In an effort to extract the relevant information, the angles are binned into equally spaced bins of $\pi / 3$. Additionally, as a rotation of $\phi=2 \pi$ brings the principle axes back to an orientation identical to that seen when $\phi=0$, the $2 \pi$ bin has been incorporated into the 0 bin.

A similar difference in the distributions of $\mathbf{n}$ is seen in those of $\phi$. Large changes from the crosswind configuration are not seen until nearly the centerline in the passive grid crossflow cases, while the region of activity is extended further above the centerline in the active grid cases. The region well above the centerline, in all cases is dominated by rotations of $2 \pi / 3$ and $4 \pi / 3$, an oscillation about $\pi$.

To better access the bulk behavior of the residence of the axis of rotation $\mathbf{n}$ and the angle of rotation $\theta$, further binning is carried out. Four areas of interest are identified in the flow, their boundaries defined by the centerline, the formulation of which was discussed in detail in section 5.2, and the shear layers on both the windward and lee sides of the jet. The location and behavior of the shear layer itself is a complex subject, the physics in portions of the shear layer mirror that of a skewed mixing layer, the sources of hanging vortices [33]. The windward side shear layer is the source of the ring vortices, which contribute vorticity to the counter rotating vortex pair [99]. Physically speaking the shear layer is an area of the flow field where the jet flow and the crossflow interact, this interaction is complex and occurs across a region which varies in thickness across the flow field. Various methods exist for approximating the trajectory of the shear layer such as the determining the loci of inflections points and 
the maxima of fluctuation amplitude or the limits of the potential core [100] [101]. The axial distribution of $\overline{u^{\prime} v^{\prime}}$ can also identify shear layer trajectory and generally the far edge of the shear layer with respect to the jet centerline as it has been observed that at the extents of the jet interaction with the crossflow, $\overline{u^{\prime} v^{\prime}} \rightarrow 0$ which is also confirmed in these data as can be seen in figures 5.13 and 5.14 [24]. This allows for the definition of the shear layers depicted as the boundaries between zone 1 (Z1) and zone 2 (Z2) on the windward side and zone 3 (Z3) and zone 4 (Z4) on the lee side as the loci of points where ${\overline{u^{\prime} v^{\prime}}}^{2} \leq 0.01{\overline{u^{\prime} v^{\prime}}}^{2}$ along the axial profiles centered on the centerline.

Figure 5.15 defines the four zones identified in the flow. Zone 1 (Z1) is the area to the windward side of the windward shear layer. Zone 2 (Z2) and zone 3 (Z3) are the areas between the centerline and the windward and lee side shear layers respectively. Zone $4(\mathrm{Z} 4)$ the area to the the lee side of the lee side shear layer. The shear layer identification scheme described above should capture the majority of the crossflow / jet flow interactions within Z2. Z1 is expected to be dominated by the dynamics of the crossflow, largely independent of the interaction. Within the shear layer, Z2 experiences high shear as the crossflow is entrained and accelerated through the interaction with the higher velocity jet fluid as well as elevated vorticity associated with the shear induced roll up vortices and the counter rotating vortex pair. Below the centerline, Z3 also contains the interaction with the crossflow, though the crossflow becomes more complex on the lee side of the jet as the jet wake is created around the core which leads the the area of recirculation seen in the mean velocities very close to the jet exit in figures 5.3 and 5.5. A concentration of y-component vorticity is also known to occur along the lee side edge [32]. The fourth zone houses the complex wake dynamics and interactions with the boundary layer where wake 

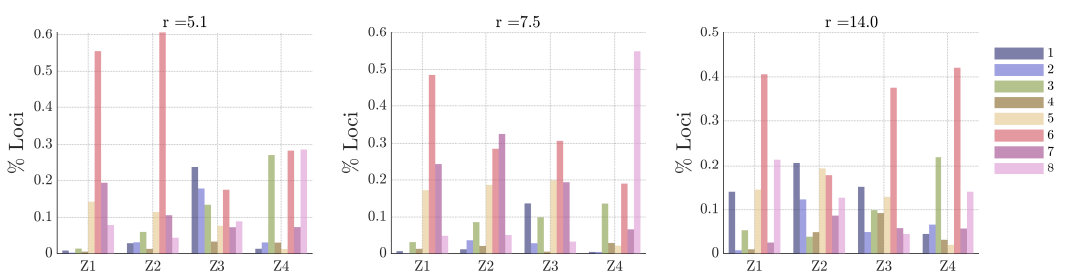

(a)
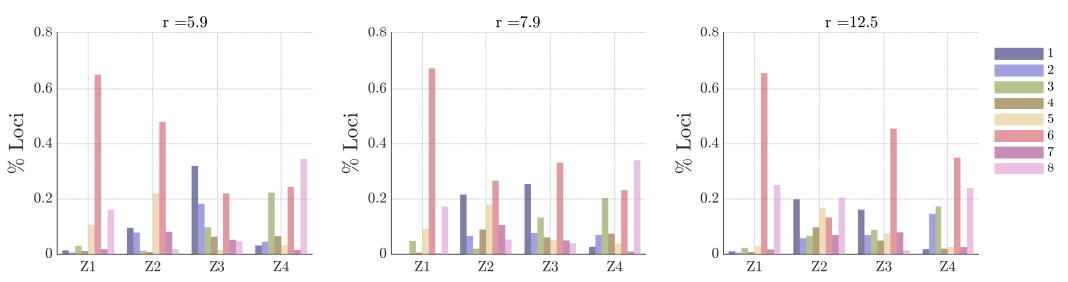

(b)

Figure 5.19: Counts of octant of $\mathbf{n}$ residence grouped by zone and normalized by the total number of queried points in each zone for both the passive grid (a) and active grid cases (b)

vortices are formed from ejections, entrained on the lee side of the jet and stretched as the jet develops downstream, vortex stretching is associated with the production of TKE [102] [103].

The residence octants of $\mathbf{n}$ and the angles of rotation $\phi$ both binned by zone and normalized by the total number of observations in each zone are found in figures 5.19 and 5.20 respectively. Focusing on the residence of $\mathbf{n}$, a residence in octant 6 is most prevalent in Z1 across all cases, again it should be noted that due to flow geometry and the constraints of the PIV window there is less of $\mathrm{Z1}$ represented in the data for the high $r$ flows. Octant 6 is the $+x,-y,-z$ octant, residence of $\mathbf{n}$ in this octant indicates that the principle axes of $b_{i j}$ are oriented down and into the page with respect to the experimental coordinate system. Interestingly, the residence octants are less distributed across $\mathrm{Z} 1$ in the active grid cases. The tighter distributions suggest that 
there is either less variation in the state of anisotropy in the more turbulent crossflow or that the effects of the jet interaction on the crossflow extend beyond the shear layer. Profiles of $F$ in figure 5.11b indicate that more isotropic turbulence seems to diffuse from the jet core to areas above the jet. The diffusion of jet core isotrtopy seen $F$ could lead to the tighter distributions in active grid $\mathrm{Z} 1$.

Crossing the windward shear layer into the jet, octant distributions in Z2 show great variation across cases. In the lowest $r$ in both the active and passive grid sets, the prevalence of octant 6 persists across the shear layer boundary into Z2. Distributions in both zones include a small but not negligible contribution from octant 1 indicating that the stresses are beginning to balance. In the intermediate values of $r$ the dominance of octant 6 is modulated by an increase in octant 7 residence, where $\mathbf{n}$ crosses the $\mathrm{z}$ axis and all components are negative. The active grid $\mathrm{Z} 2$ distribution is far more evenly distributed, the principle axes of $b_{i j}$ loose their preferred orientation. A similar effect is seen in the in the Z2 distributions of the highest $r$ in both the passive and active grid cases. In the windward portion of the jet the most variation in $\mathbf{n}$ occurs, this trend is stronger in the more turbulent crosswind of the active grid case.

At the extremes of $r$, very different distributions are seen in Z3, at low $r$ both the passive and active grid cases have their least preferential distributions across the octants. Both show a majority of residences in octants 1,2 , and 6 , three of the four octants making up the positive $\mathrm{x}$ half of the coordinate system. In the intermediate velocity ratio, the passive grid case shows a very similar distribution in Z3 as Z2. While the distributions themselves are different, a similar effect is seen in the active grid case, where octants 1 and 2 are dominant across both Z2 and Z3. Across all 

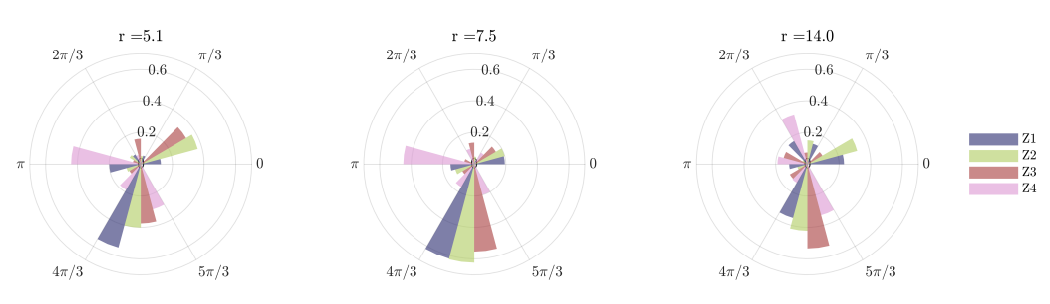

(a)
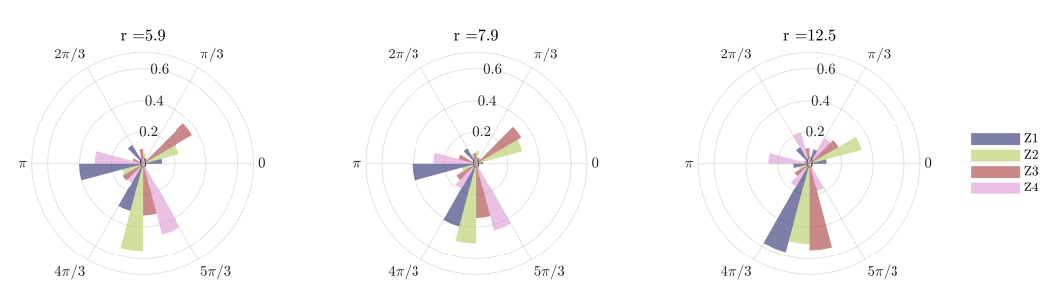

(b)

Figure 5.20: Angle of rotation $\theta$ colored binned by zone. Passive grid cases (a) and active grid cases (b)

cases considered Z4 shows largely similar behavior with the most dominant octants being 2, 6, and 8. From the contours in figure 5.17, it is clear that octant 8 is dominant near the wall and downstream across all cases. This result shows that the bulk wake dynamics are largely unaffected by the crossflow turbulence. While differences in ejection frequency, and thus the spacing of wake vortices show some dependence on $r$, the general structure of the wakes are similar in all jets in crossflow [20]. It seems that the similarities in wake structures is seen in the principle axes of $b_{i j}$, and the crossflow turbulence does not have a meaningful effect at changing these structures.

The angles of rotation, $\phi$ are displayed across the same zones in figure 5.20. The polar bar plots in figure 5.20 are constructed in such a way that the angular ticks represent the edges of the $\pi / 3$ binning scheme applied to the angles. Recall that the $5 \pi / 3-0$ bin is incorporated into the $0-\pi / 3$ bin. Within each of the wedges 
representing one bin is space for the density bars of the total counts of binned angles contained within the bounds of each of the four zones, their location within the bin does not indicate finer angular resolution, all of the angles displayed are discrete with $\pi / 3$ spacing.

The distributions show a general preference towards the $4 \pi / 3-5 \pi / 3$ range across all cases. This preference is most prevalent in Z1, Z2, and Z3. The $4 \pi / 3-5 \pi / 3$ range contains the rotation of $3 \pi / 2$ which in the rotating coordinate system where each axis is equidistant from $\mathbf{n}$ results in the $+x$ axis aligning with the $-y$ axis. The distribution of angles between zones is nearly identical across the two lower velocity ratios in the active grid set. Progressing to the high velocity ratio, Z1 residence between $\pi$ and $4 \pi / 3$ is lost to an increase in the $4 \pi / 3-5 \pi / 3$ bin. The Z4 angles become nearly evenly distributed across bins in the $r=12.5$ active grid case. Z3 shows similar behavior across the two lower active grid cases and the $r=5.1$ passive grid case with a preferential residence in the $0-\pi / 3$ bin.

In a bulk sense both the residence octants of figure 5.19 and the angles of rotation of figure 5.20 show the greatest similarity in zone 4 , the wake region. This indicates that the stresses in the wake vortex system is minimally affected by the crossflow turbulence. The evolution of invariant function $F$ support this observation. The radial profiles of $F$ shown in figure 5.11 show that in both the passive and active sets maintain moderate values of $F$ on the lee side of the jet. The similarity in $F$ seen in the wake region between passive and active grid sets is in contrast to the substantial differences in values of $F$ seen on the windward side of the jet. The residence octants of figure 5.19 show substantial differences between passive and active grid sets in Z2, the areas between the centerline and the windward shear layer. This area is where 
increased isotropy in the active grid is observed with both increased values $F$ in figures 5.10 and 5.11 and a departure from the positive $x i$ axisymmetric line on the Lumley triangles of figure 5.12. The contours of residence octants in figure 5.17 shows that the passive grid cases show frequent fluctuations between octants 5, 6, and 7 in the crossflow region as well as approaching the centerline, behavior also clearly seen in the similarities between the distributions of octants in zones 1 and 2 in figure 5.19. Active grid cases have principle axis residence in the same octants, though the fluctuation between octants is less frequent in the crossflow area, and octants 1 and 2 become active near the centerline. An examination of the behavior of the principle axes of the Reynolds stress tensor wind turbines shows that areas of the flow with more rotation about the vertical axis are the same areas where turbulence production is increased and the Reynolds stress tensor tends towards a more one component state [69]. These observations agree with what is observed on the windward edge of the jet in this work, the more frequent change in residence octants is associated with increased anisotropy which is both a cause and a result of the production of turbulence [93]. Elevated turbulence intensity in the crossflow distributes the principle axes rotations more evenly, particularly in the jet core which is consistent with the turbulence transferring energy between components via flux rather than a the production of turbulence via energy from the mean flow. 
The impact of a turbulent crossflow on the jet is considered through examining two sets of experiments. The first consists of three jets to crossflow ratios ranging from $r=5.1$ to $r=14$ with a crossflow subjected to a passive grid. The second set of experiments adds additional turbulence through the active turbulence generating grid, a set of three velocity ratios between $r=5.9$ and $r=14$ are considered. The two sets of experiments have similar enough velocity ratio ranges to make pairwise comparisons on the influence of the turbulence.

Mean streamwise flow fields, the direction of the crossflow, show a region of recirculation in the immediate wake of the jet consistent with previous studies [85]. The more turbulent cases show this recirculation damped, it is both weaker and over a smaller area. In the wall normal direction, that of the jet flow, the mean flow field shows a core of jet velocity emanating into a field of mostly zero $\bar{v}$. In the cases with greater turbulence, this core is dissipated closer to the jet exit than the passive grid cases.

Anisotropy of the Reynolds stress tensor, indicated by the function $F$, ranging from zero in isotropic turbulence to unity in pure 2 dimensional anisotropic turbulence shows dramatic differences between the highly turbulent cases and the less turbulent 
cases. All the cases considered show an area of increased isotropy in the core of the jet that shows some diffusion down towards the wall on the windward side of the jet. In the low turbulence cases the lee side edge marks a sharp decline in $F$ towards zero. The high turbulence cases the region of nearly isotropic turbulence diffuses beyond the shear layers and makes the crossflow region more isotropic. The effect is more pronounced at lower velocity ratios and grows as the flow progresses downstream of the jet exit.

The invariants of the normalized Reynolds stress anisotropy tensor reveal more information as to the nature of the anisotropy than the single parameter of $F$. Through an examination of the invariants plotted on Lumley triangles the differences in the anisotropic character of the jets in the two different type of crossflow are able to be examined in more detail. In all of the cases considered the points on the Lumley triangles move lower in $\eta$, away from more two component turbulence and towards more three dimensional turbulence as the flow progresses downstream. This is consistent with expectations in the flow, at the jet exit the flow is dominated by the strong jet flow and the developed crossflow, as the flow progresses downstream strong vortices form causing an increase in out of plane motions such as the wake vortices and the counter rotating vortex pair, the latter of which is found to be generally confined by the shear layers [32]. Lumley triangles of the passive grid cases show that points in the windward wake region tend to reside along the axisymmetric line, with a limited number of points within the showing more three dimensionality. This trend towards three dimensionality within the jet is stronger at stations farther from the jet exit. Outside the jet shear layer the passive grid cases are highly anisotropic, with invariant values indicating a single strong component driving the turbulent motions in the crossflow region. 
The higher turbulence cases have anisotropic profiles similar to the low turbulence set near the jet exit. Near four jet diameters downstream a substantial amount more of the core of the jet than in show invariant values indicating a higher degree of isotropy. The areas in the crossflow that are considered do not reach as extreme levels of one component dominated turbulence as was seen in the low turbulence case. Since the profiles near the exit are largely similar, it is a reasonable conclusion that higher turbulence in the crossflow, through its interaction with the jet, generates a more three dimensional isotropic turbulence. It is unclear if the diffusion of the isotropy above the jet indicated in figure 5.10 of the contour plots of $F$ is generated within the core of the jet and transported out, or if the interaction of the slower and less rotational crosswind with the faster and highly rotational jet flow generates more isotropic turbulence at the interface. There is an argument for the latter in that the jet typically entrains the surrounding fluid rather than expelling the jet fluid into the crossflow [104].

A complete, though low resolution view of the eigenvectors, or principle axes of the normalized anisotropy tensor via a quaternion representation of the rotation in the matrix of eigenvectors is presented. The quaternion is represented graphically through the residence octant of the three dimensional vector, $\mathbf{n}$ made up of three components of the quaternion defining an axis of rotation, and an angle, $\phi$ given by the fourth quaternion component. Low turbulence cases show a more homogeneous distribution of rotation axes, $\mathbf{n}$ in the crossflow region and an increase in in rotation activity only occurs just to the lee side of the centerline carrying on to through the windward shear layer. Beyond the shear layer the high turbulence cases do not show increased rotation in $\mathbf{n}$. Though within the jet there is more rotation compared to 
the passive grid, particularly in the intermediate velocity ratio. The wake region is remarkably similar.

The distribution of rotation angles shows the majority of the flow field is represented by rotations between $4 \pi / 3$ and $5 \pi / 3$ across both the high and low turbulence cases. The wake region is also represented by the same angles across all cases. Little effect from the turbulence can be seen in the angle of rotation.

Component budgets of the Reynolds stress anisotropy show the effect of the distribution of components on $F$. Both the low and high turbulence sets start with clean distributions of the components close to the jet exit, the distribution of components that occurs on the windward side of the jet drives $F$ towards zero as the components evolve into a more anisotropic state. This happens almost universally across the low and high turbulence sets. The notable exception being in the intermediate velocity ratios in low and high turbulence at $x^{\prime} / d=3$ and $x^{\prime} / d=6$ the value of $F$ does not drop off as in all the other cases, while the distributions of components are quite different. This suggests some dependence on $r$ is also driving the behavior of the anisotropy.

The dependence on crossflow turbulence on the level and distribution of anisotropy in the Reynolds stress tensor of the jet in cross flow is quite clear from the qualitative assessment of the data. This further complicates the modeling challenges associated with the flow at higher turbulence levels as the region of increased isotropy is ill defined. In lower crossflow turbulence regimes the lee side shear layer acts as a boundary between an anisotropic portion of the flow and the isotropic portion within 
the jet. Models could be parameterized such that different Reynolds stress treatments are used depending on the location in the flow. 


\section{Bibliography}

${ }^{1}$ T. J. Aubry, G. Carazzo, and A. M. Jellinek, "Turbulent Entrainment Into Volcanic Plumes: New Constraints From Laboratory Experiments on Buoyant Jets Rising in a Stratified Crossflow: Turbulent Entrainment in Volcanic Plumes", Geophysical Research Letters 44, 10, 198-10, 207 (2017).

${ }^{2}$ V. H. Chu and M. S. T. Abdelwahed, "Shore Attachment of Buoyant Effluent in Strong Crossflow", Journal of Hydraulic Engineering 116, 157-175 (1990).

${ }^{3} \mathrm{P}$. Ajersch, "Detailed measurements on a row of jets in a crossflow: With applications" (University of British Columbia, 1995).

${ }^{4}$ R. J. Goldstein, "Film cooling", in Advances in heat transfer, Vol. 7, edited by T. F. Irvine and J. P. Hartnett (Elsevier, 1971), pp. 321-379.

${ }^{5}$ D. G. Bogard and K. A. Thole, "Gas Turbine Film Cooling", Journal of Propulsion and Power 22, 249-270 (2006).

${ }^{6}$ J. L. Beveridge, Bow Thruster Jet-Flow, 3281 (Naval Ship Research and Development Center, Department of the Navy, Washington D.C., Mar. 1970), p. 55.

${ }^{7}$ M. Eslamian, A. Amighi, and N. Ashgriz, "Atomization of Liquid Jet in HighPressure and High-Temperature Subsonic Crossflow", AIAA Journal 52, 13741385 (2014).

${ }^{8}$ S. A. Little, K. D. Stolzenbach, and R. P. V. Herzen, "Measurements of plume flow from a hydrothermal vent field", Journal of Geophysical Research: Solid Earth 92, 2587-2596 (1987).

${ }^{9}$ C. Fisher, K. Takai, and N. Le Bris, "Hydrothermal Vent Ecosystems", Oceanography 20, 14-23 (2007).

${ }^{10}$ A. J. Findlay, E. R. Estes, A. Gartman, M. Yücel, A. Kamyshny, and G. W. Luther, "Iron and sulfide nanoparticle formation and transport in nascent hydrothermal vent plumes", Nature Communications 10, 1597 (2019).

${ }^{11}$ Y. Lou, Z. He, and X. Han, "Transport and Deposition Patterns of Particles Laden by Rising Submarine Hydrothermal Plumes", Geophysical Research Letters 47, e2020GL089935 (2020). 
${ }^{12}$ C. H. Bosanquet and J. L. Pearson, "The Spread of Smoke and Gases from Chimneys", Transactions of the Faraday Society 32, 1249 (1936).

${ }^{13}$ A. F. Rupp, S. E. Beall, L. P. Bornwasser, and D. F. Johnson, Dilution of Stack Gases in Cross Winds (Clinton National Laboratory, Oak Ridge, Tenn., 1944), p. 21.

${ }^{14} \mathrm{R}$. Margason J, The path of a jet directed at large angles to a subsonic free stream, Technical Note TN D-4919 (National Aeronautics and Space Adminisration, Langley Reserch Center, 1968).

${ }^{15}$ E. F. Hasselbrink and M. G. Mungal, "Transverse jets and jet flames. Part 2. Velocity and OH field imaging", Journal of Fluid Mechanics 443 (2001).

${ }^{16}$ L. K. Su and M. G. Mungal, "Simultaneous measurements of scalar and velocity field evolution in turbulent crossflowing jets", Journal of Fluid Mechanics 513, $1-45$ (2004).

${ }^{17}$ S. Muppidi and K. Mahesh, "Study of trajectories of jets in crossflow using direct numerical simulations", Journal of Fluid Mechanics 530, 81-100 (2005).

${ }^{18}$ J. F. Keffer and W. D. Baines, "The round turbulent jet in a cross-wind", Journal of Fluid Mechanics 15, 481 (1963).

${ }^{19} \mathrm{~J}$. Andreopoulos and W. Rodi, "Experimental investigation of jets in a crossflow", Journal of Fluid Mechanics 138, 93 (1984).

${ }^{20}$ T. F. Fric and A. Roshko, "Vortical structure in the wake of a transverse jet", Journal of Fluid Mechanics 279 (1994).

${ }^{21}$ S. H. Smith and M. G. Mungal, "Mixing, structure and scaling of the jet in crossflow", Journal of Fluid Mechanics 357, 83-122 (1998).

${ }^{22}$ J. W. Shan and P. E. Dimotakis, "Reynolds-number effects and anisotropy in transverse-jet mixing", Journal of Fluid Mechanics 566, 47 (2006).

${ }^{23}$ E. E. Callaghan, Investigation of the Penetration of an Air Jet Directed Perpendicularly to an Air Stream (National Advisory Committee for Aeronautics, 1948), 20 pp.

${ }^{24}$ D. Crabb, D. F. G. Duraõ, and J. H. Whitelaw, "A Round Jet Normal to a Crossflow", Journal of Fluids Engineering 103, 142-153 (1981).

${ }^{25}$ J. E. Broadwell and R. E. Breidenthal, "Structure and mixing of a transverse jet in incompressible flow", Journal of Fluid Mechanics 148, 405-412 (1984).

${ }^{26}$ J. Foust and D. Rockwell, "Structure of the jet from a generic catheter tip", Experiments in Fluids 41, 543 (2006).

${ }^{27}$ A. O. Demuren, "Modeling jets in cross flow", Aug. 1, 1994. 
${ }^{28}$ A. Demuren, "Numerical calculations of steady three-dimensional turbulent jets in cross flow", Computer methods in applied mechanics and engineering 37, 309-328 (1983).

${ }^{29}$ R. Claus, "Numerical calculation of subsonic jets in crossflow with reduced numerical diffusion", in 21st Joint Propulsion Conference (July 8, 1985).

${ }^{30}$ S. Arunajatesan and C. W. S. Bruner, Evaluation of Two-Equation RANS Models for Simulation of Jet-in-Crossflow Problems, SAND2011-9103C (Sandia National Laboratories, Albuquerque, NM, 2011).

${ }^{31}$ J. Ray, S. Lefantzi, S. Arunajatesan, and L. Dechant, "Bayesian Parameter Estimation of a k- Model for Accurate Jet-in-Crossflow Simulations", AIAA Journal 54, 2432-2448 (2016).

${ }^{32} \mathrm{~S}$. Muppidi and K. Mahesh, "Two-dimensional model problem to explain counterrotating vortex pair formation in a transverse jet", Physics of Fluids 18, 085103 (2006).

${ }^{33}$ L. L. Yuan, R. L. Street, and J. H. Ferziger, "Large-eddy simulations of a round jet in crossflow", Journal of Fluid Mechanics 379, 71-104 (1999).

${ }^{34}$ M. J. Woodhouse, A. J. Hogg, J. C. Phillips, and J. C. Rougier, "Uncertainty analysis of a model of wind-blown volcanic plumes", Bulletin of Volcanology 77 (2015).

${ }^{35}$ J.-L. Wu, H. Xiao, and E. Paterson, "Physics-informed machine learning approach for augmenting turbulence models: A comprehensive framework", Physical Review Fluids 3, 074602 (2018).

${ }^{36}$ J.-L. Wu, R. Sun, S. Laizet, and H. Xiao, "Representation of stress tensor perturbations with application in machine-learning-assisted turbulence modeling", Computer Methods in Applied Mechanics and Engineering 346, 707-726 (2019).

${ }^{37}$ S. R. Bistafa, "On the development of the Navier-Stokes equation by Navier", Revista Brasileira de Ensino de Física 40 (2017).

${ }^{38}$ O. Darrigol, Worlds of flow: a history of hydrodynamics from the Bernoullis to Prandtl (Oxford University Press, Oxford ; New York, 2005), 356 pp.

${ }^{39}$ H. Schlichting, Boundary layer theory, Seventh (McGraw Hill, 1979).

${ }^{40}$ S. Rodriguez, Applied Computational Fluid Dynamics and Turbulence Modeling: Practical Tools, Tips and Techniques (Springer International Publishing, Cham, 2019).

${ }^{41}$ D. G. Fox and D. K. Lilly, "Numerical simulation of turbulent flows", Reviews of Geophysics 10, 51-72 (1972).

${ }^{42}$ C. D. Argyropoulos and N. C. Markatos, "Recent advances on the numerical modelling of turbulent flows", Applied Mathematical Modelling 39, 693-732 (2015). 
${ }^{43}$ W. C. Reynolds, "The potential and limitations of direct and large eddy simulations", in Whither Turbulence? Turbulence at the Crossroads, Vol. 357, edited by J. L. Lumley, Lecture Notes in Physics (Springer Berlin Heidelberg, Berlin, Heidelberg, 1990), pp. 313-343.

${ }^{44}$ O. Reynolds, "IV. On the dynamical theory of incompressible viscous fluids and the determination of the criterion", Philosophical Transactions of the Royal Society of London. (A.) 186, 123-164 (1895).

${ }^{45}$ W. K. George, "Lectures in Turbulence for the 21st Century", Imperial College of London, 2013.

${ }^{46}$ R. Stull, "Turbulence Closure Techniques", in An Introduction to Boundary Layer Meteorology, Vol. 13, Atmospheric Sciences Library (Springer, Dordrecht, 1988).

${ }^{47}$ J. Boussinesq, Essai sur la théorie des eaux courantes (L'Academie Des Sciences De L'Institut De France, 1877).

${ }^{48}$ F. G. Schmitt, "About Boussinesq's turbulent viscosity hypothesis: historical remarks and a direct evaluation of its validity", Comptes Rendus Mécanique 335, 617-627 (2007).

${ }^{49}$ D. C. Wilcox, Turbulence Modeling for CFD, Third (DCW Industries, La Canada, California, 2006).

${ }^{50}$ S. B. Pope, Turbulent flows (Cambridge university press, 2000).

${ }^{51}$ M. S. Uberoi, "Effect of Wind-Tunnel Contraction on Free-Stream Turbulence", Journal of the Aeronautical Sciences 23, 754-764 (1956).

${ }^{52}$ J. L. Lumley and G. R. Newman, "The return to isotropy of homogeneous turbulence", Journal of Fluid Mechanics 82, 161 (1977).

${ }^{53}$ S. Sarkar and C. G. Speziale, "A simple nonlinear model for the return to isotropy in turbulence", Physics of Fluids A: Fluid Dynamics 2, 84-93 (1990).

${ }^{54}$ B. Perot and C. Chartrand, "Modeling return to isotropy using kinetic equations", Physics of Fluids 17, 035101 (2005).

${ }^{55}$ T. Sjögren and A. V. Johansson, "Development and calibration of algebraic nonlinear models for terms in the Reynolds stress transport equations", Physics of Fluids 12, 1554-1572 (2000).

${ }^{56}$ J. Rotta, "Statistiche Theory micht-homogener Turbulenz, 1. Mitteilung", Zeitschrift fuer Physik 129, 547-572 (1951).

${ }^{57}$ K.-S. Choi and J. L. Lumley, "The return to isotropy of homogeneous turbulence", Journal of Fluid Mechanics 436 (2001).

${ }^{58}$ C. G. Speziale, "Analytical Methods for the Development of Reynolds-Stress Closures in Turbulence", Annual Review of Fluid Mechanics 23, 107-157 (1991). 
${ }^{59}$ U. Schumann, "Realizability of Reynolds-stress turbulence models", Physics of Fluids 20, 721 (1977).

${ }^{60}$ J. O. Hinze and P. J. H. Builtjes, "Rotation of the Reynolds stress tensor in a decaying gridgenerated turbulent flow", The Physics of Fluids 20, S175-S178 (1977).

${ }^{61}$ R. P. Paul, Robot manipulators: mathematics, programming, and control: the computer control of robot manipulators (MIT Press, 1981).

${ }^{62}$ H.-L. Pham, V. Perdereau, B. V. Adorno, and P. Fraisse, "Position and orientation control of robot manipulators using dual quaternion feedback", in 2010 IEEE/RSJ International Conference on Intelligent Robots and Systems (Oct. 2010), pp. 658663.

${ }^{63}$ E. Pervin and J. A. Webb, Quaternions in computer vision and robotics (CarnegieMellon Univ., Dept of Computer Science, Pittsburg, PA, 1982).

${ }^{64}$ J. C. K. Chou and M. Kamel, "Finding the Position and Orientation of a Sensor on a Robot Manipulator Using Quaternions", The International Journal of Robotics Research 10, 240-254 (1991).

${ }^{65}$ J. Diebel, "Representing Attitude: Euler Angles, Unit Quaternions, and Rotation Vectors", 2006.

${ }^{66}$ K. Symon, Mechanics, 2nd ed. (Addison-Wesley Publishing Co., 1960).

${ }^{67} \mathrm{~K}$. W. Spring, "Euler parameters and the use of quaternion algebra in the manipulation of finite rotations: A review", Mechanism and Machine Theory 21, 365-373 (1986).

${ }^{68}$ H. Goldstein, C. P. Pool Jr, and J. L. Safko, Classical Mechanics, 3rd (Pearson, 2002).

${ }^{69}$ N. Hamilton and R. B. Cal, "Anisotropy of the Reynolds stress tensor in the wakes of wind turbine arrays in Cartesian arrangements with counter-rotating rotors", Physics of Fluids 27, 015102 (2015).

${ }^{70}$ E. Mitchell and A. Rogers, "Quaternion Parameters in the Simulation of a Spinning Rigid Body", SIMULATION 4, 390-396 (1965).

${ }^{71}$ J. Stuelpnagel, "On the Parametrization of the Three-Dimensional Rotation Group", SIAM Review 6, 422-430 (1964).

${ }^{72}$ W. R. Hamilton, Elements of Quaternions, edited by W. E. Hamilton, Cambridge University Press Reprint 2009 (Longmans, Green, \& Co, London, 1866).

${ }^{73} \mathrm{~K}$. Shoemake, "Animating rotation with quaternion curves", in Proceedings of the 12th annual conference on Computer graphics and interactive techniques, SIGGRAPH '85 (July 1, 1985), pp. 245-254. 
${ }^{74}$ NASA, Onboard Navigation Systems Characteristics, Technical Memorandum (National Aeronautics and Space Adminis- tration, Mission Planning and Analysis Division, Houston, TX, Mar. 1979), p. 316.

${ }^{75}$ R. Müller and M. Vette, "Comparison of practically applicable mathematical descriptions of orientation and rotation in the three-dimensional Euclidean space", in Tagungsband des 3. Kongresses Montage Handhabung Industrieroboter, edited by T. Schüppstuhl, K. Tracht, and J. Franke (2018).

${ }^{76}$ J. C. Hart, G. K. Francis, and L. H. Kauffman, "Visualizing quaternion rotation", ACM Transactions on Graphics 13, 256-276 (1994).

${ }^{77}$ D. M. Henderson, Euler angles, quaternions, and transformation matrices working relationships, Technical Memorandum NASA-TM-74839 (National Aeronautics and Space Administration, Mission Planning and Analysis Division, Houston, TX, July 1, 1977), p. 42.

${ }^{78}$ S. W. Sheppard, "Quaternion from Rotation Matrix", Journal of Guidance and Control 1, 223-224 (1978).

${ }^{79}$ A. R. Klumpp, "Singularity-free extraction of a quaternion from a direction-cosine matrix", Journal of Spacecraft and Rockets 13, 754-755 (1976).

${ }^{80}$ R. A. Spurrier, "Comment on " Singularity-Free Extraction of a Quaternion from a Direction-Cosine Matrix"", Journal of Spacecraft and Rockets 15, 255-255 (1978).

${ }^{81}$ A. Cayley, "Recherches Ultérieures sur les Déterminants gauches", in The Collected Mathematical Papers (Cambridge University Press, Cambridge, 1889).

${ }^{82}$ S. Sarabandi, A. Perez-Gracia, and F. Thomas, "Singularity-free computation of quaternions from rotation matrices in E4 and E3", in Applied Geometric Algebra in Computer Science and Engineering (2019), pp. 109-115.

${ }^{83}$ H. Makita and K. Sassa, "Active Turbulence Generation in a Laboratory Wind Tunnel", in Advances in Turbulence 3, edited by A. V. Johansson and P. H. Alfredsson (Springer Berlin Heidelberg, Berlin, Heidelberg, 1991), pp. 497-505.

${ }^{84}$ B. Wieneke, "PIV uncertainty quantification from correlation statistics", Measurement Science and Technology 26, 074002 (2015).

${ }^{85}$ W. Graf and B. Yulistiyanto, "Experiments on flow around a cylinder; the velocity and vorticity fields", Journal of Hydraulic Research 36, 637-654 (1998).

${ }^{86}$ L. Cortelezzi, A. R. Karagozian, et al., "On the formation of the counter-rotating vortex pair in transverse jets", Journal of Fluid Mechanics 446, 347-373 (2001).

${ }^{87} \mathrm{~K}$. Mahesh, "The Interaction of Jets with Crossflow", Annual Review of Fluid Mechanics 45, 379-407 (2013).

${ }^{88}$ S. Corrsin, "I. Extended applications of the hotwire anemometer. II. Investigations of the flow in round, turbulent jets", PhD thesis (California Institute of Technology, 1947). 
${ }^{89}$ S. Dey, P. Paul, S. Zeeshan Ali, and E. Padhi, "Reynolds stress anisotropy in flow over two-dimensional rigid dunes", Proceedings of the Royal Society A: Mathematical, Physical and Engineering Sciences 476, 20200638 (2020).

${ }^{90}$ O. Özcan, K. E. Meyer, and P. S. Larsen, "Measurement of mean rotation and strain-rate tensors by using stereoscopic PIV", Experiments in Fluids 39, 771-783 (2005).

${ }^{91}$ M. J. Lee, J. Kim, and P. Moin, "Structure of turbulence at high shear rate", Journal of Fluid Mechanics 216, 561-583 (1990).

${ }^{92}$ T. Uzkan and W. C. Reynolds, "A shear-free turbulent boundary layer", Journal of Fluid Mechanics 28, 803-821 (1967).

${ }^{93}$ J. H. Ferziger and S. Shaanan, "Effect of anisotropy and rotation on turbulence production", Physics of Fluids 19, 596 (1976).

${ }^{94}$ K. E. Meyer, J. M. Pedersen, and O. öZcan, "A turbulent jet in crossflow analysed with proper orthogonal decomposition", Journal of Fluid Mechanics 583, 199 (2007).

${ }^{95}$ S. S. Lu and W. W. Willmarth, "Measurements of the structure of the Reynolds stress in a turbulent boundary layer", Journal of Fluid Mechanics 60, 481 (1973).

${ }^{96}$ M. Farano, S. Cherubini, J.-C. Robinet, and P. De Palma, "Optimal bursts in turbulent channel flow", Journal of Fluid Mechanics 817, 35-60 (2017).

${ }^{97}$ M. M. Madden, "Octant Analysis of The Reynolds Stresses in the Three Diemensional Turbulent Boundary Layer of a Prolate Spheroid" (Virginia Polytechnic Institute and State University, Blacksburg, Virginia, July 24, 1997), 213 pp.

${ }^{98}$ R. Fearn and R. P. Weston, "Vorticity Associated with a Jet in a Cross Flow", AIAA Journal 12, 1666-1671 (1974).

${ }^{99}$ R. M. Kelso, T. T. Lim, and A. E. Perry, "An experimental study of round jets in cross-flow", Journal of Fluid Mechanics 306, 111 (1996).

${ }^{100}$ S. Megerian, J. Davitian, L. S. D. B. Alves, and A. R. Karagozian, "Transverse-jet shear-layer instabilities. Part 1. Experimental studies", Journal of Fluid Mechanics 593, 93-129 (2007).

${ }^{101}$ D. R. Getsinger, C. Hendrickson, and A. R. Karagozian, "Shear layer instabilities in low-density transverse jets", Experiments in Fluids 53, 783-801 (2012).

${ }^{102}$ S. Gopalan, B. M. Abraham, and J. Katz, "The structure of a jet in cross flow at low velocity ratios", Physics of Fluids 16, 2067-2087 (2004).

${ }^{103}$ P. A. Davidson, Turbulence: An Introduction for Scientists and Engineers (Oxford University Press, 2015), 647 pp.

${ }^{104}$ S. Muppidi and K. Mahesh, "Direct numerical simulation of round turbulent jets in crossflow", Journal of Fluid Mechanics 574, 59 (2007). 
${ }^{105}$ L. Prandtl, "Bericht fiber untersuchungen zur ausgebildeten turbulent.(eng. trans. in report on investigation on developed turbulence, naca-tm 1231)", Zeitschrift für angewandte Mathematik und Mechanik 5 (1925).

${ }^{106}$ J. Smagorinsky, "General Circulation Experiments with the Primative Equations: I. The Basic Experiment", Monthly Weather Review 91, 99-164 (1963).

${ }^{107}$ A. Kolmogorov, "Equations of Turbulent Motion of an Incompressible Fluid", trans. by D. B. Spalding, Izv. Akad. Nauk SSSR, Seria Fizicheska VI, 56-58 (1942).

${ }^{108}$ G. Alfonsi, "Reynolds-Averaged NavierStokes Equations for Turbulence Modeling", Applied Mechanics Reviews 62 (2009).

${ }^{109}$ B. E. Launder and D. B. Spalding, "The numerical computation of turbulent flows", in Numerical prediction of flow, heat transfer, turbulence and combustion (Elsevier, 1983), pp. 96-116. 


\section{Appendix}

\section{A $\quad$ Turbulence Viscosity Modeling}

\section{A.1 Zero Equation Models}

Zero-equation models prescribe the scales which define $\nu_{T}$ algebraically. The first example of a zero-equation model is the mixing-length theory of Prandtl [105], which is based on an analogy between the mean free path in the kinetic theory of gases and a turbulent length scale. The mixing-length theory defines

$$
\nu_{T}=l_{m}^{2}\left|\frac{d \bar{u}}{d y}\right|
$$

where $l_{m}$ is the mixing length, which near a plane wall is defined as $l_{m}=\kappa y$, where $\kappa$ is the von Kármán constant when the flow is plane shear and the only mean velocity component is parallel to the wall and varies with distance normal to the wall [58]. When applied to the boundary layer flow the Reynolds shear stress becomes

$$
\overline{u^{\prime} v^{\prime}}=\rho \kappa^{2} y^{2}\left(\frac{d u}{d y}\right)^{2} .
$$


With the additional assumption that the shear stress is constant near the wall, the "law of the wall" is the result and is given by

$$
u^{+}=\frac{1}{\kappa} \ln y^{+}+C
$$

where $u^{+}=\bar{u} / u_{\tau}$ and $y^{+}=y u_{\tau} / \nu$ with $u_{\tau}$ the friction velocity and $\mathrm{C}$ a dimensionless constant. The "law of the wall" is able to quite accurately predict the velocity profile of the turbulent boundary layer [39].

Beyond Prandtls model for the boundary layer, zero-equation models can be extended to three dimensional flows by by applying the length scale to the full strain rate tensor. Such a model is proposed by Smagorinsky [106] setting the turbulent viscosity as

$$
\nu_{T}=l_{m}^{2}\left(\bar{S}_{i j} \bar{S}_{i j}\right)^{1 / 2}
$$

There remains a necessity with the mixing length model to apply a length scale, the choice of which requires prior knowledge of the flow, or very general assumptions, often times both. Despite their limitations, they are often used today to supplement a more robust models at small scales. The Smagorinsky model is used to resolve sub-grid scales in large eddy simulation, and the law of the wall is often used as a wall function in $k-\mathcal{E}$ models.

\section{A.2 Kinetic Energy Models}

The premise of kinetic energy models originates with a theory introduced by Kolmogorov [107], a velocity scale relates to the kinetic energy as

$$
u^{*}=c k^{1 / 2}
$$


This relation initially gave rise to a family of one equation models to close the transport equation for the kinetic energy [50],

$$
\begin{aligned}
& \frac{D k}{D t}=\frac{\partial k}{\partial t}+\bar{u} \cdot \nabla k \\
& =-\nabla \cdot \mathbf{T}^{\prime}+\mathcal{P}-\mathcal{E},
\end{aligned}
$$

Where $D k / D t$ is the material derivative of the turbulent kinetic energy, $\mathbf{T}^{\prime}$, the flux, $\mathcal{P}$, the production, and $\mathcal{E}$, the dissipation. The kinetic energy models resolves the fields for $\bar{u}, p, l_{m}, k, \nu_{T}$, and $\overline{u_{i}^{\prime} u_{j}^{\prime}}$. One equation models require modeling of the flux and dissipation terms, to achieve this empirical length scales are again required [108].

Eliminating the prescribed length scale Launder and Spalding [109] propose that the length scale be related to both the kinetic energy and the dissipation

$$
l=C_{D} k^{3 / 2} / \mathcal{E}
$$

The elimination of the prescribed length scale requires that an additional transport equation be solved for the dissipation. Rather than the exact transport equation for the dissipation, an empirical model is used as the exact transport equation operates at dissipation scales which are beyond the reach of the $k-\mathcal{E}$ models [50]. The empirical form of the dissipation transport equation is

$$
\frac{D \mathcal{E}}{D t}=\nabla \cdot\left(\frac{\nu_{t}}{\sigma_{\mathcal{E}}} \nabla \mathcal{E}\right)+C_{\mathcal{E} 1} \frac{\mathcal{P} \mathcal{E}}{k}-C_{\mathcal{E} 2} \frac{\mathcal{E}^{2}}{k}
$$

The only term that must be modeled is the flux term from (A.6). The the turbulent viscosity becomes entirely a function of $k$ and $\mathcal{E}$,

$$
\nu_{T}=C_{\mu} k^{2} / \mathcal{E}
$$

Leaving three empirical constants to be determined experimentally. 